Document downloaded from:

http://hdl.handle.net/10251/158176

This paper must be cited as:

Torregrosa, AJ.; Gil, A.; Quintero-Igeño, P.; Ammirati, A.; Denayer, H.; Desmet, W. (2019). Prediction of flow induced vibration of a flat plate located after a bluff wall mounted obstacle. Journal of Wind Engineering and Industrial Aerodynamics. 190:23-39.

https://doi.org/10.1016/j.jweia.2019.04.008

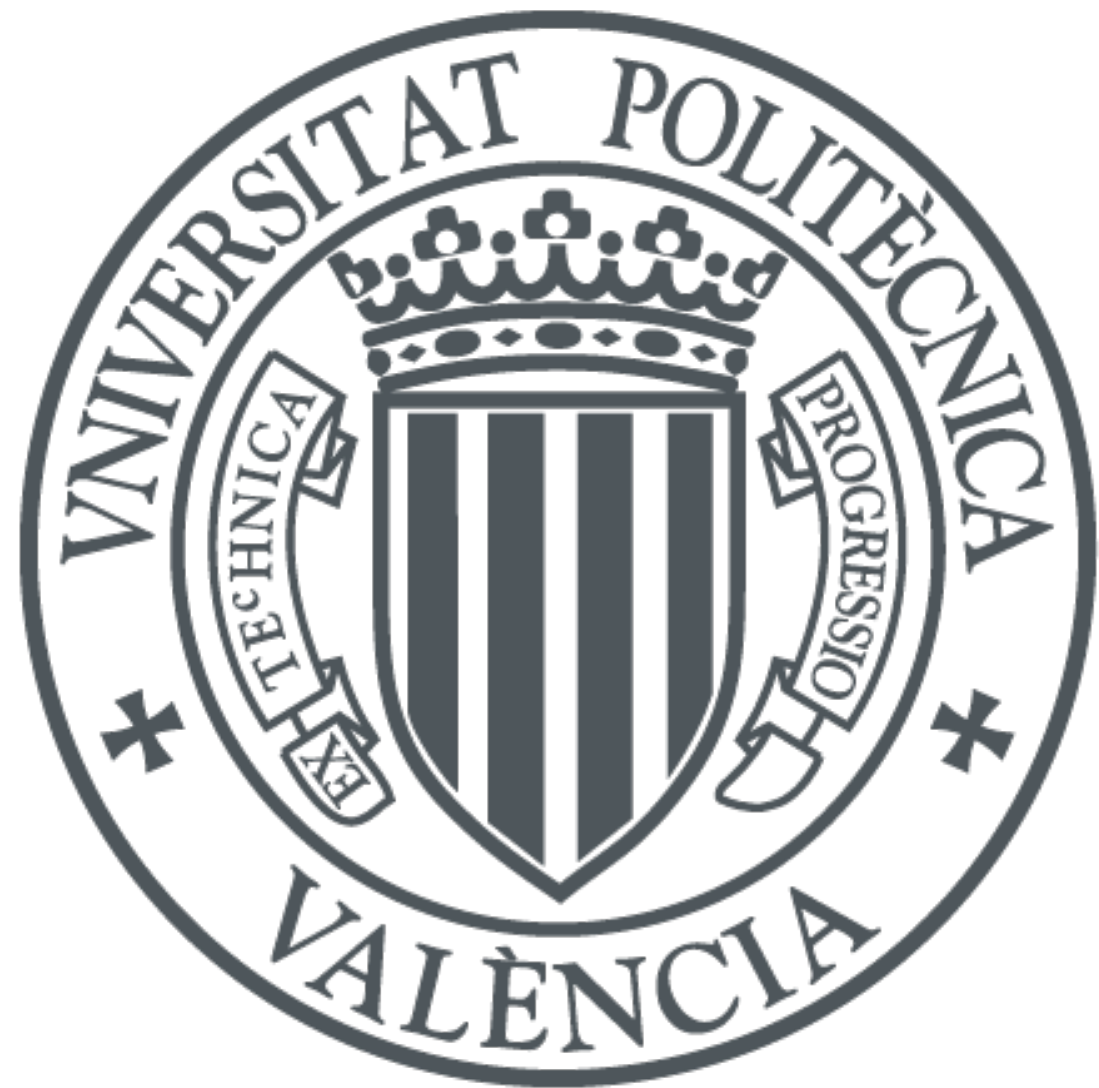

The final publication is available at

https://doi.org/10.1016/j.jweia.2019.04.008

Copyright Elsevier

Additional Information 


\title{
Prediction of Flow Induced Vibration of a flat plate located after a bluff wall mounted obstacle
}

\author{
A. Torregrosa, A. Gil, P. Quintero* \\ CMT-Motores Térmicos, Universitat Politècnica de València, Camino de Vera s/n, 46022 Valencia, Spain. \\ A. Ammirati, H. Denayer, W. Desmet \\ ${ }^{a}$ Department of Mechanical Engineering, KU Leuven, Celestijnenlaan 300, B-3001 Heverlee, Belgium \\ ${ }^{b}$ DMMS Lab, Flanders Make, Belgium
}

\begin{abstract}
Accurate prediction of Flow Induced Vibration phenomena is currently a field of major interest due to the use of lightweight materials in the automotive and aerospace industry. This article studies the turbulent flow around a wallmounted obstacle, and the induced deformations produced by the pressure fluctuations on a plate located downstream the obstacle. The methodology used is a combination of experimental tests and numerical simulations. On one side, experiments were carried out in a wind tunnel test facility equipped with Particle Image Velocimetry to characterize the fluid velocity field, and laser vibro-meter to measure the vibrations of the plate. On the other side, Fluid-Structure Interaction (FSI-one-way) has been calculated by considering different turbulence modelling approximations (RANS and LES). Finally, numerical results have been analyzed and validated against the experiments in terms of main flow structures and the vibroacoustic response of the plate.
\end{abstract}

Keywords: Flow Induced Vibration, one way coupling, coupling, Navier Stokes, Fluid Structure Interaction, Large Eddy Simulation, Reynolds Averaged Navier Stokes

\section{Introduction}

During the past decades, because of use of increasingly lightweight materials for the construction of components in direct contact with a moving fluid, the accurate prediction of flow-structure interactions (FSI) has become a topic of primal interest. Aerospace industry has traditionally been one of the main drivers for research about these phenomena. For instance, it is well known that a wing subjected to certain values of the flow velocity can experience inadmissible static deformations (divergence, see Hilderbrand and Reissner [1]) or periodic time-increasing oscillations (flutter, see Bisplinghoff et al. [2] or Jeong and Kwon [3]) which can lead to mechanical static or fatigue failures.

In this sense, wide number of important engineering problems are related with the flow around bluff bodies. Therefore, the accurate prediction of FSI phenomena over the own body or the vibration of structures located at its wake is of major interest. Special mention could be given to the work of Schewe and Larsen [4], who used wind tunnel measurements in order to predict the loads acting over a bluff bridge deck cross section; Augier et al. [5] performed a tight coupled simulation of the FSI phenomena appearing at yacht sails subjected to a turbulent flow. More recently, Zhang et al. [6] performed Detached Eddy Simulations (DES) in order to predict the broadband frequency content of the displacements generated at a high building excited by a turbulent fluid flow supposing one way coupling. This work was later expanded by Ricci et al. [7], solving the flow with Large Eddy Simulations (LES).

In the automotive industry, due to the significantly lower working velocities compared with those expected on aircraft applications, these phenomena less often lead to a tight-high displacement fluid structure interaction (FSI).

\footnotetext{
*Corresponding author. Tel.: +34 96 3877650, fax: +34963877659.
}

Email address: pedquiig@mot.upv .es (P. Quintero ) 
However, understanding and being able to predict FSI is of growing interest. It is well known that NVH (noise, vibration and harshness) is becoming a crucial topic of study for automotive manufacturers. Because of stricter regulations and increasing customer expectations, a major mechanism for the generation of undesired aerodynamic noise inside the cabin is the excitation of the structure by the turbulent flow around the vehicle. This fact, in combination with the development of increasingly silent engines, explains the increasing interest on FSI also in automotive applications.

One of the first investigations in this field was carried out by Davies [8] who studied the excitation of a flat plate under a turbulent boundary layer (TBL) using modal analysis. Later, more efforts have been directed to obtain a correct prediction of this as, for instance, in the works of Graham [9], Howe [10] or Frampton [11]. However, their assumptions are of difficult application when the flow is dominated by a highly non-isotropic turbulence, as is the case for the flow downstream of a wall-mounted obstacle, where the large structures containing the energy of the flow should be accounted for, and therefore modeled.

A particularly interesting example can be found at the vibrations appearing under the body of a moving car. In fact, the turbulent unsteady flow generates a fluctuating pressure which acts on the floor of the vehicle. This fluctuating pressure induces vibrations of the floor, which are responsible for radiating noise to the interior of the cabin. Related with this, some interesting studies can be found in the literature. For instance, Springer et al. [12] numerically studied a simplified version of the problem: a fluid flow passes over a wall-mounted obstacle which generates a turbulent wake, and the unsteady pressure fluctuations after the wake excite a thin flat plate which starts to vibrate. However, in their work they did not provide experimental data about the plate vibration; they over predicted the reattachment length by more than $50 \%$ and a low number of structural modes were excited which, in principle, disagrees with experiments [13].

Mueller et al. [13] investigated both the structural vibration and acoustic behavior of flat plates of different materials and sizes under different excitations. They showed how, for the case were the flow becomes turbulent, most of the natural modes of vibration of the plate are excited which, as could be expected, corresponds to a peak in the Sound Pressure Level spectrum for the corresponding natural frequencies. Schafer et al. [14] investigated the same simplified geometry as [12], using a very thin flat plate of $40 \mu \mathrm{m}$, and assuming two-way fluid structure coupling, obtaining accurate results for the flow field, compared with the experiments. However, the prediction of the induced vibration agreed only qualitatively with the experiments. The main factor underlying the deviations between numerical and experimental results could be the low plate stiffness, which leads to large displacements, which are indeed difficult to predict by using a linear structural model. Furthermore, a plate with a value of structural stiffness so low, cannot be considered as representative for most of the cases related with NVH.

The present work intends to complement the works of [14] and [12]. A similar simplified geometry was considered, where a steel plate of $0.5 \mathrm{~mm}$ thick is located just downstream of the obstacle. Both the fluid domain and the structural vibration are numerically modelled, making use of different numerical models, and the results are compared with experiments. It is shown that a one way interaction model provides accurate results for both the turbulent fluid flow and the flow-induced vibrations of the plate. The mechanism exciting each vibration mode is analyzed in detail. The capabilities of different turbulence models, such as the Large Eddy Simulation (LES) or the, computationally less demanding, Reynolds Averaged Navier Stokes (RANS) are explored and their applicability to the prediction of the flow field and the plate deformation is assessed.

It will be shown how a one way interaction model can provide accurate results for both the turbulent fluid flow and the flat plate flow-induced vibrations. The mechanism exciting each vibration mode will be analyzed. Capabilities of mean flow turbulence models, as Reynolds Averaged Navier Stokes (RANS) will be explored and it will be shown that they can provide acceptable values for the time averaged values of the plate deformation, even when some important assumptions are introduced in the computation of the fluid flow. The followed methodology can be summarized as follows. First, the flow field was computed both by using LES and RANS for different grid refinement and compared with the available experimental data; at the same time, the structural model of the plate was validated by comparing computational and experimental eigenfrequencies and eigenvectors. When both the flow and structural model are validated, they are coupled using a one way methodology by mapping the CFD pressure solution onto the FEM structural mesh. Finally, the flow induced vibrations are calculated using this structural model and analyzed, comparing the response at different frequencies with the previously computed eigenfrequencies. Figure 1 shows an schematic overview of this workflow. in order to ease the interpretation of the current work. 


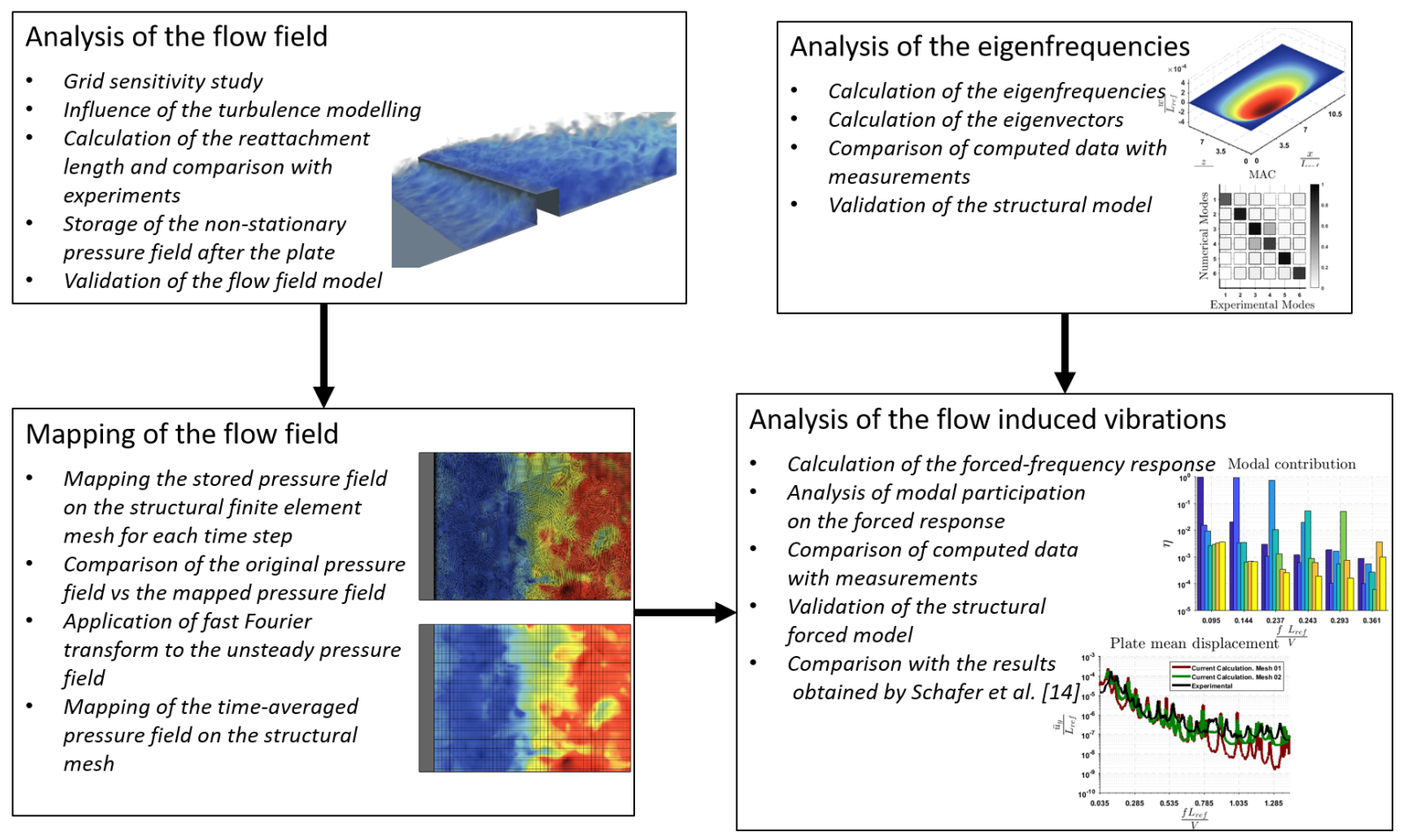

Figure 1: Diagram of the working flow used during the current work

The paper is structured as follows: First, Section 2 introduces the case studied, and the numerical tools used to solve both the fluid flow and the structural response is provided. Section 3 analyses the theoretical background of the numerical methods used for the current investigation. Then, Section 4 describes the main features of the experimental facilities used and the tests performed to validate the numerical results. Section 5 discusses the main results of this study, comparing LES, RANS and experiments, and discussing the differences observed between each case. Finally, in Section 6 summarizes the most important results and conclusions.

\section{Description of the test case}

In this investigation, a simplified under-body flow is modeled which, nevertheless, can be considered as representative for most of the relevant physical phenomena. The configuration consists of (1) a channel which walls are considered rigid; (2) a rigid square step which forces a turbulent flow downstream of it, dominated by large scales, and (3) a thin flexible wall where the magnitude of the displacement is sufficiently high to allow appreciable deformations (and noise radiation), but low enough to not influence the fluid field noticeably.

Figure 2 shows a sketch of the fluid domain geometry. $L_{r e f}=1.5 \mathrm{~cm}$ is the edge length of the step, which is confined in a channel of $5 L_{\text {ref }}$ in height and $10 L_{\text {ref }}$ in width, leading to a blocking ratio of 0.20 , which can be considered to be representative of blockage found on cars' underbody flows. Preliminary calculations with different values of the blockage ratio were performed, providing qualitatively similar results. In consequence, for reasons of brevity, only the blockage of 0.20 is discussed in the following.

A flow of air, with density $\rho_{\infty}=1.225 \mathrm{~kg} \mathrm{~m}^{-3}$, viscosity $\mu=1.78 \times 10^{-5} \mathrm{~Pa}$ s and average velocity $V_{\infty}=22 \mathrm{~m} \mathrm{~s}^{-1}$, which correspond to a Reynolds number $\mathrm{Re}=22710$ and a Mach number, $\mathrm{Ma}=0.06$, enters the domain from the left. From a fluid dynamic perspective, the obstacle, the plate wall and the bottom walls are treated with a nonslip boundary condition. The inlet and the outlet are located sufficiently far such that the frequency response of the variables of interest is not affected by their location.

It is expected that the boundary layer at the upper wall is sufficiently thin such that its effects on the vibrational response of the plate can be neglected. Therefore, in order to keep the computational cost affordable, a slip boundary 
condition was used for this wall. For the lateral walls, a similar reasoning could be given, and a periodicity condition can be stated, in a similar way as in the work of Schafer et al. [14] or David et al. [15].

The plate is mounted just after the step and covers the whole width of the channel, with a length of $40 / 3 L_{r e f}$. The edges parallel to the flow direction are clamped, while the contours perpendicular to the flow are mounted in a simply supported manner. The plate is made of steel, with Young's Modulus of $E=200 \mathrm{GPa}$; density of $\rho_{s}=7745 \mathrm{~kg} \mathrm{~m}^{-3}$; Poisson's ratio equaling $v=0.35$ and a thickness of $h=0.5 \mathrm{~mm}$.

Slip wall

\section{Flow direction}

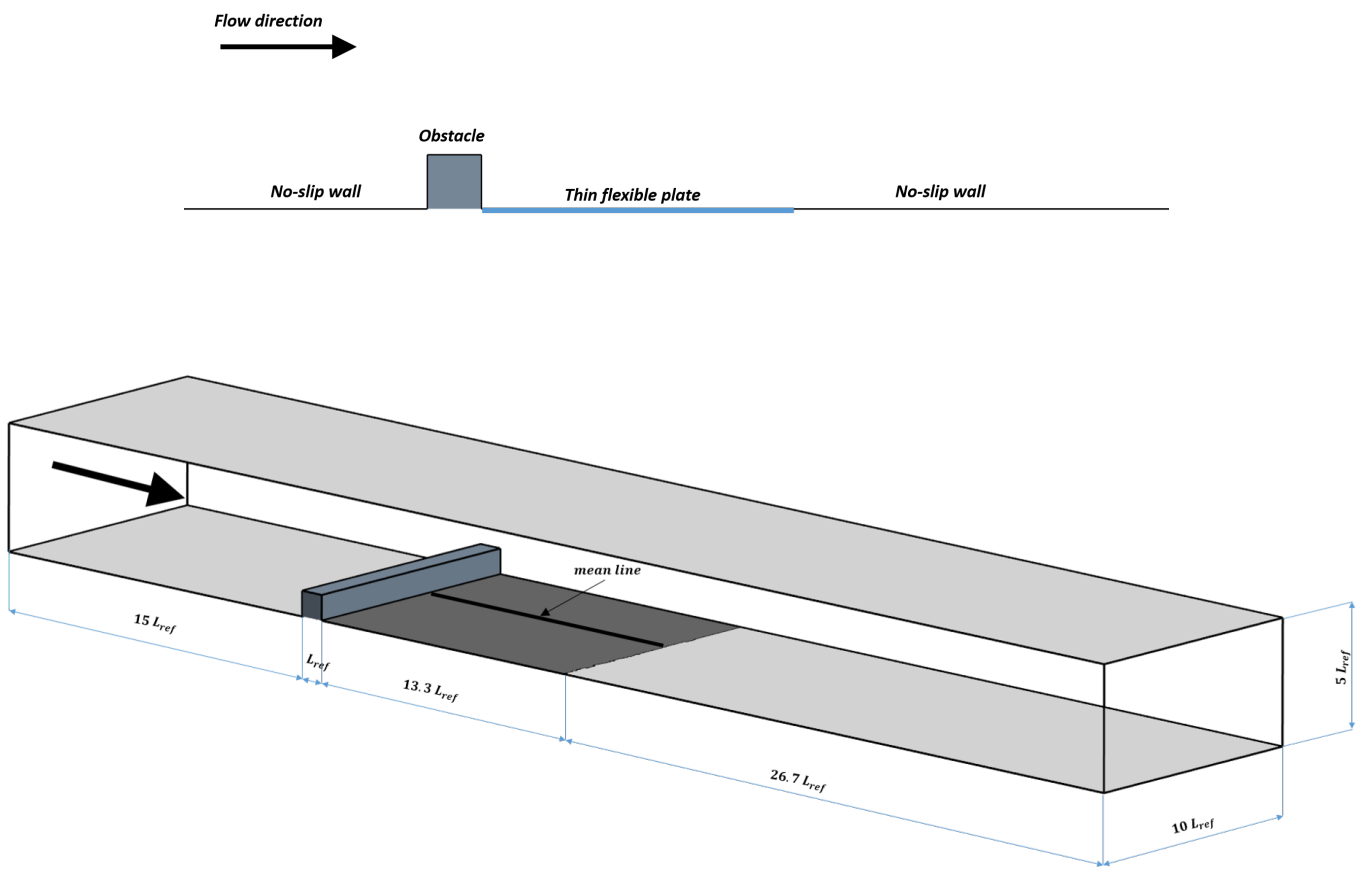

Figure 2: Fluid domain geometry sketch (not scale)

\section{Numerical methodology}

\subsection{Thin plate structural model}

As stated, all walls except the plate are considered infinitely rigid and their displacements can thus be neglected. In order to predict the deformations at the plate induced by the fluid flow, the equations for an elastic solid are applied to this body. However, it is well known that, if the plate is sufficiently thin, those equations reduce to the equation of a Kirchoff-Love plate, [16], [17], which is shown in Equation 1. In this paper, this equation will be used to describe the structural part, since $h / L_{r e f}=1 / 30$.

$$
\frac{E h^{3}}{12\left(1-v^{2}\right)}\left(\frac{\partial^{4} u}{\partial x^{4}}+2 \frac{\partial^{4} u}{\partial x^{2} \partial z^{2}}+\frac{\partial^{4} u}{\partial z^{4}}\right)+\rho_{s} h \frac{\partial^{2} u}{\partial t^{2}}=p
$$

Here, $x$ and $z$ are, respectively, the directions parallel and perpendicular to the flow; $u$ is the normal displacement of the plate (in the $y$ direction) and $p$ is the pressure acting on the structure. Note that, regardless the strength of the coupling, the plate will be influenced by the flow forces by means of this last term, as indicated, among others, by Bathe and Zhang [18]. In order to investigate the relative importance of the different parameters on the response of the 
plate, Equation 1 can be non-dimensionalized and transformed into the frequency domain, as expressed in Equation 2:

$$
\left(\frac{\partial^{4} u^{*}}{\partial x^{*}}+2 \frac{\partial^{4} u^{*}}{\partial x^{*^{2}} \partial z^{*^{2}}}+\frac{\partial^{4} u^{*}}{\partial z^{*}}\right)-\frac{m^{*}}{k^{*}} S t^{2} u^{*}=\frac{C_{p}}{k^{*}}
$$

Here, $k^{*}=\frac{E}{6 \rho_{\infty} V_{\infty}^{2}\left(1-v^{2}\right)}\left(\frac{h}{L_{r e f}}\right)^{3}=2254$ is the non-dimensional stiffness parameter, representing the relative importance of the elastic and pressure forces acting on the plate; $m^{*}=8 \pi^{2} \frac{h}{L_{r e f}} \frac{\rho_{0}}{\rho_{\infty}}=16640$ is the non-dimensional mass, which represents the relationship between the solid and fluid inertia; $C_{p}=\frac{p}{\frac{1}{2} \rho_{\infty} V_{\infty}^{2}}$ is the pressure coefficient, and $\mathrm{St}=\frac{f L_{r e f}}{V_{\infty}}$ is the Strouhal number. Both the displacements and the coordinates have been non-dimensionalized with the length of the obstacle leading to $u^{*}=\frac{u}{L_{\text {ref }}}$ and $\left(x^{*}, y^{*}, z^{*}\right)=\left(\frac{x}{L_{\text {ref }}}, \frac{y}{L_{\text {ref }}}, \frac{z}{L_{\text {ref }}}\right)$.

It should be pointed out that, when $\mathrm{St}=0$, Equation (2) can be used to predict the stationary time-averaged deformation of the plate.

When the plate is not excited by a fluid flow (e.g. the fluid velocity is zero), Equation (2) reduces to an eigenvalue problem, as indicated in Equation 3. As expected, in that case the response becomes completely independent from the flow velocity:

$$
\left(\frac{\partial^{4} u^{*}}{\partial x^{*^{4}}}+2 \frac{\partial^{4} u^{*}}{\partial x^{*^{2}} \partial z^{*^{2}}}+\frac{\partial^{4} u^{*}}{\partial z^{*^{4}}}\right)-\frac{m^{*}}{k^{*}} S t^{2} u^{*}=0
$$

An inspection of Equation 2 allows to distinguish which level of FSI coupling may be expected depending on these non-dimensional numbers. It should be noted that the order of magnitude at which each kind of interaction appears depends also on the frequency content of the excitation. When the structure is excited at its resonance frequency, it will tend to experience higher deformations. The different coupling levels are characterized as follows:

- When $k^{*}>>1$ and $m^{*}>>1$, equation 2 leads to a solution with very low displacements, which will not appreciably modify the fluid domain geometry and response.

- When $k^{*}>>1$ and $m^{*}<<1$ equation 2 leads to a solution with very low displacements, but with high velocities. The fluid domain geometry will not be modified in this case, but the velocity of the wall could excite inherent instabilities on the flow, affecting its behavior.

- When $k^{*}<<1$ the pressure forces are several orders of magnitude higher than the elastic forces, so that the structure experiences deformations which are comparable to its main dimensions. Thus, the fluid domain geometry experiences changes and the interaction must be calculated in a fully coupled way.

A one-way structural excitation can be assumed for the current value of both the stiffness and mass parameters, as it will be shown later, even for frequencies near the resonance. Thus, Equation 2 was discretized and solved by the Finite Element Method, making use of the commercial code Virtual.Lab. The pressure field, obtained making use of the methodologies explained in the following sections, was mapped onto the structure mesh and used as a load boundary condition. The plate was discretized by using 50 elements at each direction.

\subsection{Large Eddy Simulation}

In order to obtain the unsteady pressure fluctuations over the wall downstream of the step, Large Eddy Simulations have been shown to provide very good agreement with experiments at low and moderate Reynolds numbers, as can be checked, for instance, in the works of Yang and J.H. [19] or Zhengtong and Castro [20]. For Large Eddy Simulations, the Navier-Stokes equations are solved over a filtered domain, as shown in Equation 4. Note how, due to the low Mach number of the flow, it is considered to be incompressible:

$$
\left\{\begin{array}{c}
\frac{\partial V_{i}}{\partial x_{i}}=0 \\
\frac{\partial V_{i}}{\partial t}+V_{j} \frac{\partial V_{i}}{\partial x_{j}}=-\frac{1}{\rho} \frac{\partial p}{\partial x_{i}}+\frac{\partial}{\partial x_{j}} \tau_{i j}
\end{array}\right.
$$


Here, $V_{i}$ and $p_{i}$ are the resolved-scale velocity and pressure, respectively; $\tau_{i j}$ is the $i j$ component of the subgridscale (SGS) Reynolds stress; $\rho_{0}$ is the fluid density, and $\mu$ is the dynamic viscosity of the fluid. In the computation performed here, an implicit filter was used, so that the grid size itself can be considered to be the filter width.

The subgrid stress tensor result from the interaction between the larger, resolved eddies and the smaller, unresolved eddies, and is modeled using the Boussineq approximation, as indicated by Equation 5:

$$
\boldsymbol{\tau}_{t}=2 \mu_{t} \mathbf{S}-\frac{2}{3}\left(\mu_{t} \nabla \cdot \vec{V}+\rho k\right) \mathbf{I}
$$

where $\mathbf{S}$ is the strain rate tensor and $k$ is the subgrid kinetic energy. In this work, the subgrid scale turbulent viscosity, $\mu_{t}$, was modeled using WALE (Wall Adapting Local Eddy Viscosity), which has been shown to be less dependent on the value of the model coefficient, $C_{w}$, than the classical Smagorinsky Subgrid Scale Model (see the works of Nicoud and Ducros [21] and Smagorinsky [22] for more information about this topic). The WALE model assumes a mixing-length type equation for the subgrid scale viscosity, as follows:

$$
\mu_{t}=\rho \Delta^{2} S_{w}
$$

being $\Delta=C_{w} \mathcal{V}^{1 / 3}$ a length scale parameter dependent of the cell volume $\mathcal{V}$, and $\mathbf{S}_{\mathbf{w}}$ is a deformation parameter, dependent of the strain rate tensor. Here, the model constant was set to $C_{w}=0.544$, given that this value has been shown to provide acceptable results both for homogeneous isotropic decaying turbulence and for channel flows (see, for example, [23] or Malloupas et al. [24]).

The equations were discretized and solved by means of the commercial software STAR-CCM+. RANS and LES computations were performed over two different meshes, with approximately $4 \times 10^{6}$ and $20 \times 10^{6}$ volume cells. The convection scheme was set to second order upwind for RANS calculations and a bounded central-differencing [25] for LES. In order to ensure reproducibility of the results, Table 1 shows the mesh size at different parts of the fluid domain. For the coarser mesh, the no-slip walls were meshed using a prism layer with total thickness of $0.012 L_{r e f}$ and 8 layers, in order to ensure that the wall $y^{+}$lies within the viscous sublayer, as will be verified later. A time step of $\frac{\Delta t V_{\infty}}{L_{r e f}}=0.050$ for the first mesh and $\frac{\Delta t V_{\infty}}{L_{r e f}}=0.025$ for the second, ensure a Courant-Friedrichs-Lewy (CFL) lower than 1 for the most part of the domain, as can be illustrated by the distribution of CFL, shown in Figure 3. A general qualitative sketch of the computational fluid mesh can be seen in Figure 4.

Table 1: Orientative dimensions of the fluid flow meshes

\begin{tabular}{|l|c|c|}
\hline Mesh Parameter & Mesh 01 & Mesh 02 \\
\hline \hline Farfield mesh size & $0.680 L_{r e f}$ & $0.400 L_{r e f}$ \\
\hline Down walls mesh size & $0.050 L_{r e f}$ & $0.030 L_{r e f}$ \\
\hline Step walls mesh size & $0.010 L_{r e f}$ & $0.006 L_{r e f}$ \\
\hline Near field mesh size & $0.050 L_{r e f}$ & $0.030 L_{r e f}$ \\
\hline Wake mesh size & $0.100 L_{r e f}$ & $0.060 L_{r e f}$ \\
\hline Number of elements & $4 \times 10^{6}$ & $20 \times 10^{6}$ \\
\hline
\end{tabular}



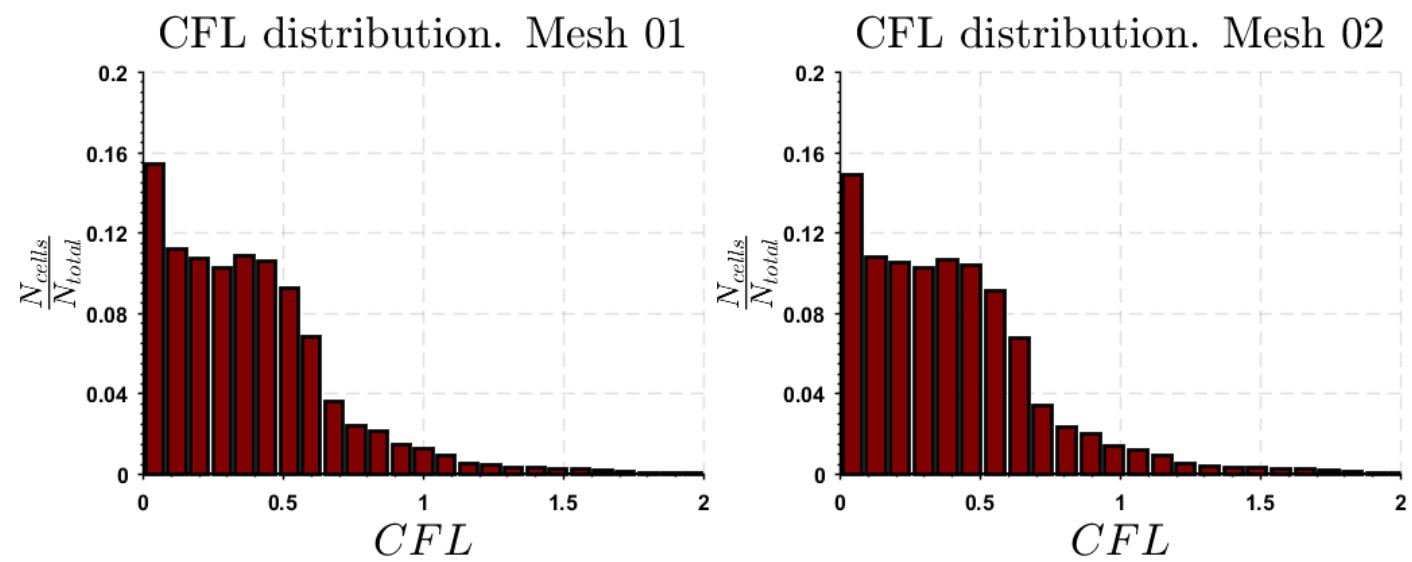

Figure 3: Ratio of CFL distribution over the entire fluid domain for the meshes of $N_{\text {elements }} \approx 4 \times 10^{6}$ (left) and $N_{\text {elements }} \approx 20 \times 10^{6}$ (right)

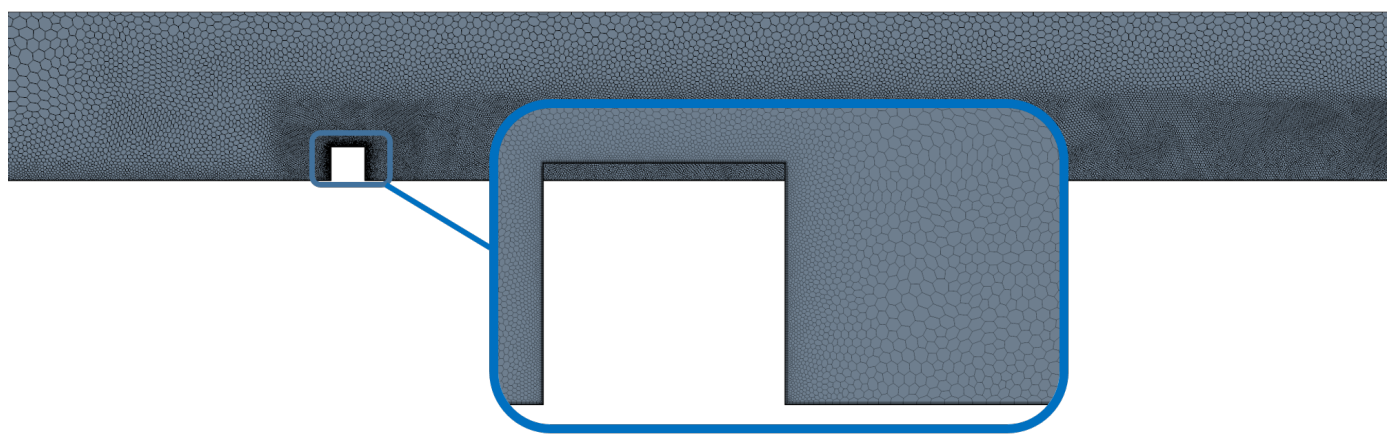

Figure 4: Sketch of the computational fluid mesh

With the mesh resolution used, it is not possible to properly model the turbulence of the inlet boundary condition and, consequently, laminar-constant velocity inflow will be considered, similarly as Schafer et al. [14]. The assumption of neglecting the free stream turbulence in the computation can be justified as follows: as the vibration response at low-to-medium frequencies is dominated by the large turbulent structures downstream of the obstacle, this effect is expected to be of second order importance, as can be verified by the works of Bearman and Morel [26] or Nakamura and Ozono [27]. For instance, at [26] it can be observed how, for free stream turbulence values below $6.1 \%$ the wall pressure distribution behind a hard-corners reward facing step and the reattachment length are only slightly affected by the variation of the turbulence intensity.

\subsection{Reynolds Averaged Navier Stokes}

The Reynolds Averaged Navier Stokes equations allows to obtain a mean flow solution, which can be used in order to compute parameters as the reatachment length or the plate static defformations. These equations can be derived from the complete set of mass, momentum and energy conservation equations, and are shown next for an incompressible flow [28].

$$
\left\{\begin{array}{c}
\frac{\partial\left\langle V_{i}\right\rangle}{\partial x_{i}}=0 \\
\frac{\partial\left\langle V_{i}\right\rangle}{\partial t}+\left\langle V_{j}\right\rangle \frac{\partial\left\langle V_{i}\right\rangle}{\partial x_{j}}=\frac{\mu}{\rho} \frac{\partial^{2}\left\langle V_{i}\right\rangle}{\partial x_{j} \partial x_{i}}-\frac{\partial\left\langle v_{i} v_{j}\right\rangle}{\partial x_{j}}-\frac{1}{\rho} \frac{\partial\langle p\rangle}{\partial x_{i}}
\end{array}\right.
$$

where $\left\langle V_{i}\right\rangle$ represents the component in the $i^{t h}$ direction of the mean velocity field, $\langle\vec{V}\rangle$ and $\langle p\rangle$ represents the ensemble average pressure field. 
Closure of equations (7) can only be achieved by modeling the terms $\left\langle u_{i} u_{j}\right\rangle$ which are commonly referred to as the Reynolds stresses. The selection of an appropriate turbulence model is of primal importance for the evaluation of the flow characteristics. In this paper, the $k-\omega$ model with shear stress transport (SST) was used with this purpose. This turbulence model has been extensively used in the literature for this type of flows, and has been shown to provide good results for the pressure coefficient near the step [29]-[30], but over predicting the length of the reattachment zone in comparison with experiments or Direct Numerical Simulation [31].

The $k-\omega$ SST model was proposed by Menter [32] and is a transitional model in which the formulation considered varies from the $k-\omega$ turbulence model proposed by Wilcox [33] in the vicinity of the walls, to the $k-\varepsilon$ model away from the walls, thus solving the main inconveniences of both models. In addition to a transport equation for the turbulent kinetic energy, $k$, the $k-\varepsilon$ and the $k-\omega$ turbulent models solve a transport equation for the turbulent dissipation rate, $\epsilon$, and the specific turbulent dissipation rate, $\omega$, respectively. These variables are related by $\omega \propto \varepsilon / k$ and allow obtaining the turbulent viscosity $v_{t}=C_{\mu} k^{2} / \varepsilon\left(C_{\mu}=0.09\right.$ being a modeling constant). This turbulent viscosity is used to model the value of the Reynolds stress tensor as:

$$
\left\langle v_{i} v_{j}\right\rangle=\frac{2}{3} \delta_{i j}-v_{T}\left(\frac{\partial\left\langle V_{i}\right\rangle}{\partial x_{j}}+\frac{\partial\left\langle V_{j}\right\rangle}{\partial x_{i}}\right)
$$

Having added the new transport equations for the resolution of the fluid flow, boundary conditions should be additionally imposed to them. For the current computation, the turbulence intensity at the inlet was set to be $I_{\text {inlet }}=$ 0.01 , with a turbulent viscosity ratio of $\frac{\mu_{T}}{\mu}=5$. Different values of these values ranging from $I_{\text {inlet }} \in[0.005-0.05]$ and $\frac{\mu_{T}}{\mu}=[1-10]$ were tried, leading to similar results. As a consequence, for reasons of brevity, only the first value indicated will be shown.

\subsection{Mapping of the fluid field pressure}

As stated, the structural model of Equation 2 will be solved assuming one way coupling model. This means that the vibrational behavior of the plate is governed by the pressure field generated by the turbulent flow, as can be observed at the right term of Equation 2. However, and due to the high value of the non dimensional stiffness parameter, $k^{*}=2254$, it will be supposed that the resulting displacements are low enough as they do not affect the behaviour of the fluid flow. Note that, at this stage, this is only a reasonable hypothesis which will only be confirmed by the comparison of the computational results with measurements.

In order to consider the influence of the pressure contribution onto the plate displacement, note that the finervolume-mesh CFD solution has to be mapped onto the grosser-finite-element FEM grid. In order to do so, while preserving the value of the nodal forces, a conservative distance mapping scheme was used, with a second order interpolation, in a similar way as explained by Ullrich and Taylor [34], [35] or Jones [36].

Figure 5 shows an example of how the mapping process works. Figure 5 (left) represents the pressure coefficient of the computed LES fluid flow at an arbitrary time step while Figure 5 (right) shows the resulting mapped pressure field which will be used for the computation of the displacements in accordance with Equation 2. Note how the results are essentially equal, even though the smallest resolved scales are filtered on the mapped mesh. However, as the small structures are the less energy containing ones [28] and, in accordance with Taylor's hypotheses they will be related with the energy contained at high frequencies [37] the mapped pressure can be considered to be accurate enough for low-medium frequencies. 

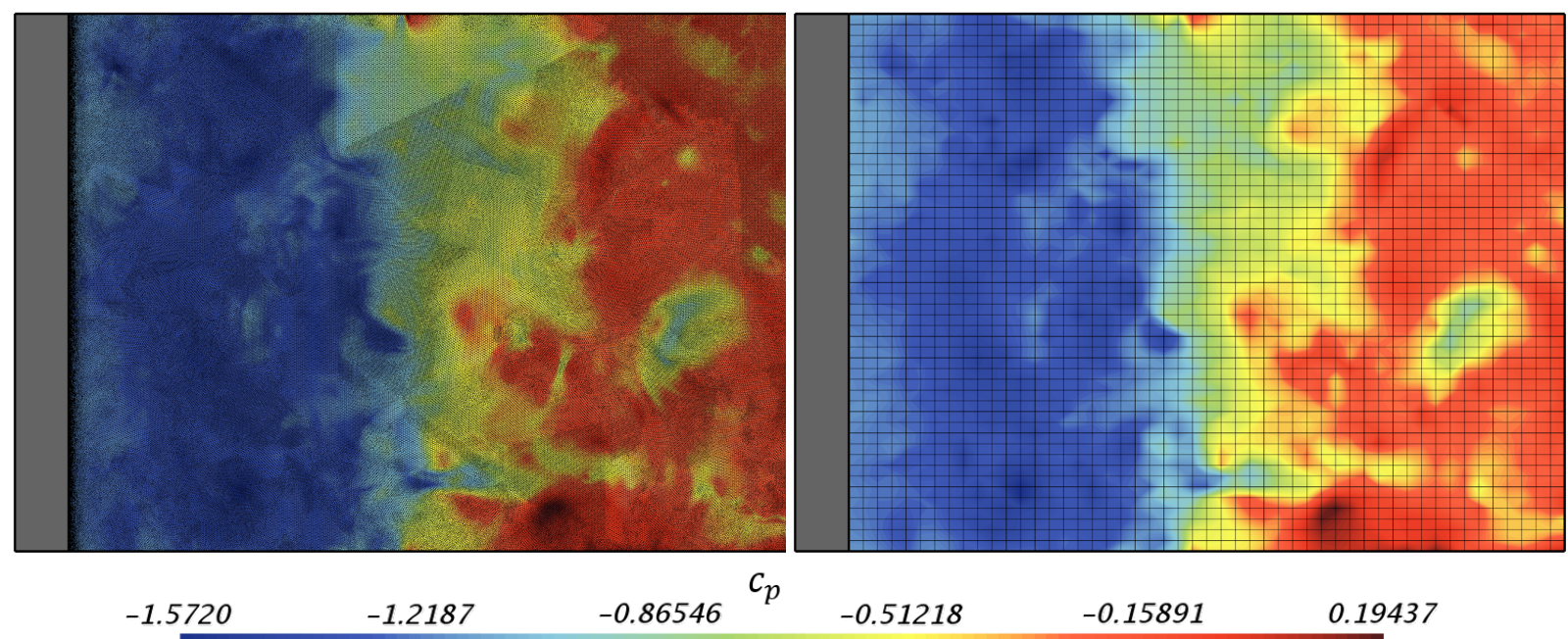

$-1.2187$

$-0.86546$

$-0.51218$

$-0.15891$

0.19437

Figure 5: Pressure coefficient distribution at the back plate for: fluid finite volume mesh (left) and structural finite element mesh (right) at an arbitrary time step of the Large Eddy Simulation solution

\section{Experimental methodology}

In order to validate the numerical model, several experimental analyses have been performed in the KU Leuven silent wind tunnel. The tunnel is generally used for identification of aeroacoustic sources and acoustic propagation mechanism in flow confined environment for the subsonic flow region. A detailed description can be found in Roeck and Desmet [38].

A roots blower is used to generate a time-uniform flow field. In order to guarantee identical inlet conditions for different measurement campaigns, a frequency regulator with PID controller, coupled with downstream pressure and flow rate sensors, is attached to the roots blower. After the roots blower, a heat exchanger is installed. The presence of the aftercooler is made necessary by the significant increase of the temperature generated by the roots blower. The high temperature generates an increase in the speed of sound which alters the acoustic propagation phenomena. Using the heat exchanger, the temperature increase is reduced with temperature fluctuations of less than $5 \%$ between different measurements.

After the heat exchanger, the flow passes an acoustic labyrinth, consisting of approximately $10 \mathrm{~m}$ of circular silencers and a dedicated designed anechoic termination. The termination has a dual purpose: on one side it reduces all the noise generated upstream acting like a sponge and ensuring an anechoic inflow. On the other side, it guarantees that the acoustic waves are not reflected back to the test section where measurements are performed in the plane wave region.

Subsequently, the flow is guided through a flow conditioner containing a divergent, screens and honeycombs sections, which allow to obtain low turbulence inlet conditions, with a turbulence intensity below $2 \%$.

A similar configuration as the one described in section 2 is used. The tunnel has a rectangular cross section of $10 L_{r e f}$ width and $5 L_{r e f}$ height. A steel plate is flush mounted right behind a step with a square cross section of $5 L_{r e f}$. The step extends over the width of the tunnel cross section. The plate is $20 \mathrm{~cm}$ long and $15 \mathrm{~cm}$ wide with a thickness of $0.5 \mathrm{~mm}$. In order to obtain a flush mounted configuration, an external frame is used. The edges of the plate parallel to the flow direction are clamped between the frame and the duct side walls. The two sides perpendicular to the flow direction are simply fixed to the frame using double-sided tape to obtain an approximately simply supported configuration. The averaged air velocity at the inlet is maintained at $V_{\infty}=22 \mathrm{~m} \mathrm{~s}^{-} 1$, with free stream turbulence intensity lower than $2 \%$. The temperature of the air is controlled in order to set a Reynolds number of approximately $R e=22710$. At first, in order to validate the plate boundary condition and to identify the modal behavior of the plate, a modal analysis on the plate for no flow condition has been performed using hammer test, as explained in Ren and De Roeck [39]. 
The vibration of the plate under operating condition is measured with a Polytec scanning vibrometer with a configuration similar to which used by Roozen et al. [40]. The acquisition is performed on 165 measurement points regularly spaced over the plate and for each of them 100 averages are performed. Finally using the Solo PIV laser (for more information, refer to the work of Butscher et al. [41]) and a high speed camera the flow characteristics are measured along the tunnel middle plane, normal to the $z$ component, at different stations along the flow propagation direction, which will allow to accurately measure the location of the end of the recirculation bubble, by the inspection of the velocity field, as will be shown later.

\section{Results and discussion}

\subsection{Analysis of the fluid flow}

One of the main objectives of the current work is to provide a good understanding of the mechanisms governing the flow-induced vibration, and to validate methodologies currently in use. With those purposes, in this section the main characteristics of the flow field are analyzed in detail by comparing LES and RANS results with the experimental measurements.

In order to ensure that the data of the current article are not dependent on the grid resolution it is recommendable to make a comparison to check that they are almost constant between each study. Thus, both time averaged and frequency content will be analyzed. First of all, the predicted forces exerted over the step were computed and analyzed. These forces are nondimensionlized in accordance with Equation 9, where $F_{y}$ and $F_{x}$ are the forces perpendicular and parallel to the main flow, respectively. Their time averaged value, $\left(<C_{D}>,<C_{L}>\right)$ and standard deviation, $\left(\left(<C_{D}^{\prime 2}>\right)^{1 / 2},\left(<C_{L}^{\prime 2}>\right)^{1 / 2}\right)$ are shown in Table 2 for the different turbulence modeling (when possible) and grid resolution.

$$
C_{L}=\frac{F_{y}}{1 / 2 \rho_{\infty} V_{\infty}^{2} L_{r e f} b} \quad C_{D}=\frac{F_{x}}{1 / 2 \rho_{\infty} V_{\infty}^{2} L_{r e f} b}
$$

Table 2: Comparison of the force coefficients between RANS and LES computations for the different mesh resolutions

\begin{tabular}{|l|c|c|c|c|}
\cline { 2 - 5 } \multicolumn{1}{c|}{} & $<C_{D}>$ & $\left(<C_{D}^{\prime 2}>\right)^{1 / 2}$ & $<C_{L}>$ & $\left(<C_{L}^{\prime 2}>\right)^{1 / 2}$ \\
\hline \hline LES. Mesh 01 & 2.173 & 0.023 & 1.344 & 0.033 \\
\hline RANS. Mesh 01 & 2.062 & - & 1.214 & - \\
\hline LES. Mesh 02 & 2.170 & 0.028 & 1.435 & 0.037 \\
\hline RANS. Mesh 02 & 2.103 & - & 1.238 & - \\
\hline
\end{tabular}

Note how, for the prediction of the time averaged force coefficients, the RANS computations can provide with results with a difference of a $5 \%$ and $14 \%$ for the prediction of the average horizontal and vertical force coefficients, respectively. Moreover, the LES computation predicts small variations of these coefficients around the average value, letting deduce that RANS can provide acceptable results for those coefficients both quantitatively and qualitatively. Both the mesh of $N_{\text {element }} \approx 4 \times 10^{6}$ and $N_{\text {element }} \approx 20 \times 10^{6}$ provide similar results for the same turbulence modeling. Note how the temporal standard deviation of the variables for the finer mesh tends to higher values, suggesting a higher energy content at medium-high frequencies.

This can be confirmed by Figure 6, that shows the time domain response (left) and the frequency content (right) of these forces. This last curve was calculated applying the fast Fourier transform to the temporal history of the variables. The sampling length was taken in a way that the terms of the Fourier series were not substantially changed when adding new samples. It can be observed that no dominant frequencies exist, and that the spectral content quickly decays for St $>0.35$. Additionally, note how the frequency content obtained by the finer mesh is higher, especially at the range of medium high frequencies. 

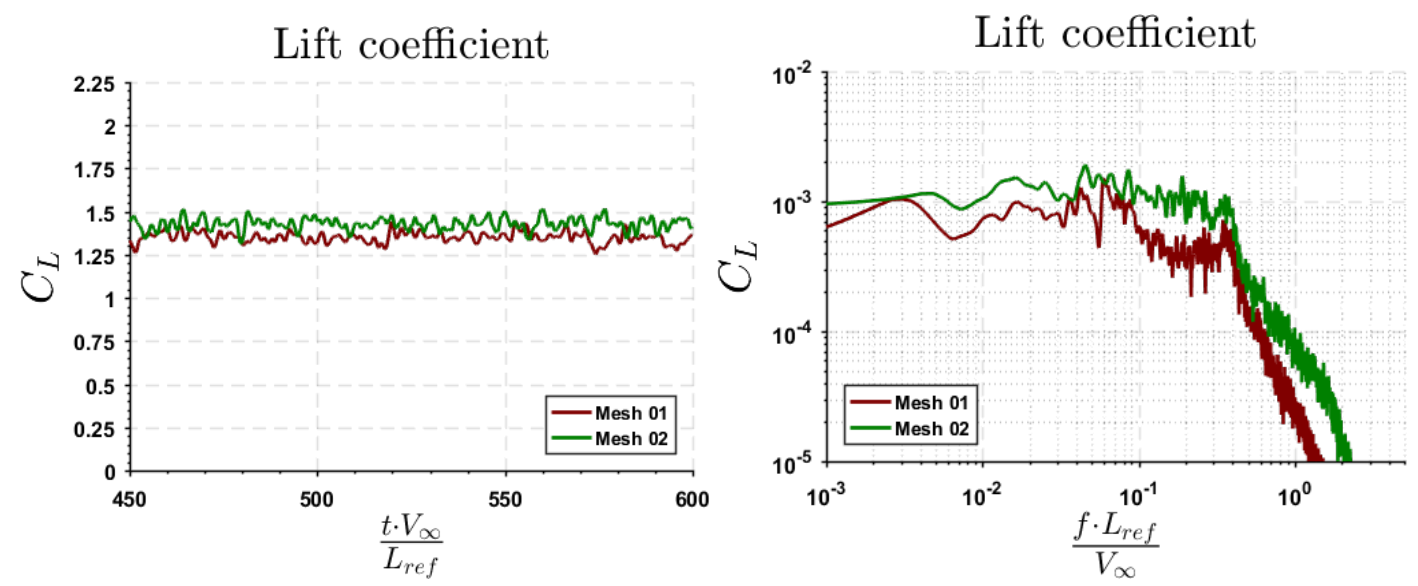

Figure 6: Force time history (left) and frequency content (right). Computation for the mesh with $N_{\text {elements }} \approx 20 \times 10^{6}$

Other result which is considered of importance in order to evaluate the predictive capabilities of RANS and LES for the different meshes is the reattachment length. The reattachment length is measured using PIV, which allows to obtain the vectors of the velocity field at a zone near to the location where the reattachment is expected to lie in, as shown in Figure 7 where an example of the time-averaged field is sketched. The location of the reattachment length was defined as the place at which the velocity vectors near the down wall tend to be parallel to the ground (with an angle of less than $0.5 \mathrm{deg}$ ), with the same direction as the incoming fluid flow. This zone is represented by the vertical line of the Figure 7 and it was found to be at $\left(x_{\text {reattach }} / L_{\text {ref }}\right)_{\text {exp }} \approx 10 \pm 0.7$, measured from the end of the step.

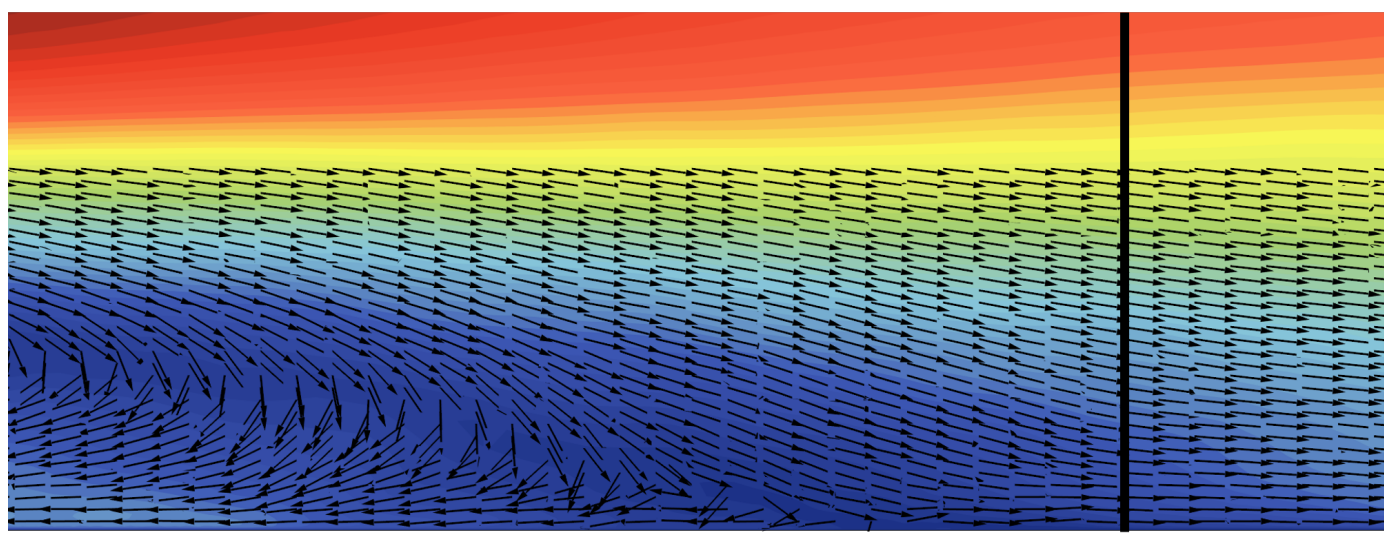

reattachment line

0.00000

0.32600

0.65200

0.97800

1.3040

1.6300

Figure 7: Vectors of time averaged velocity for the identification of the experimetnal reattachment length 


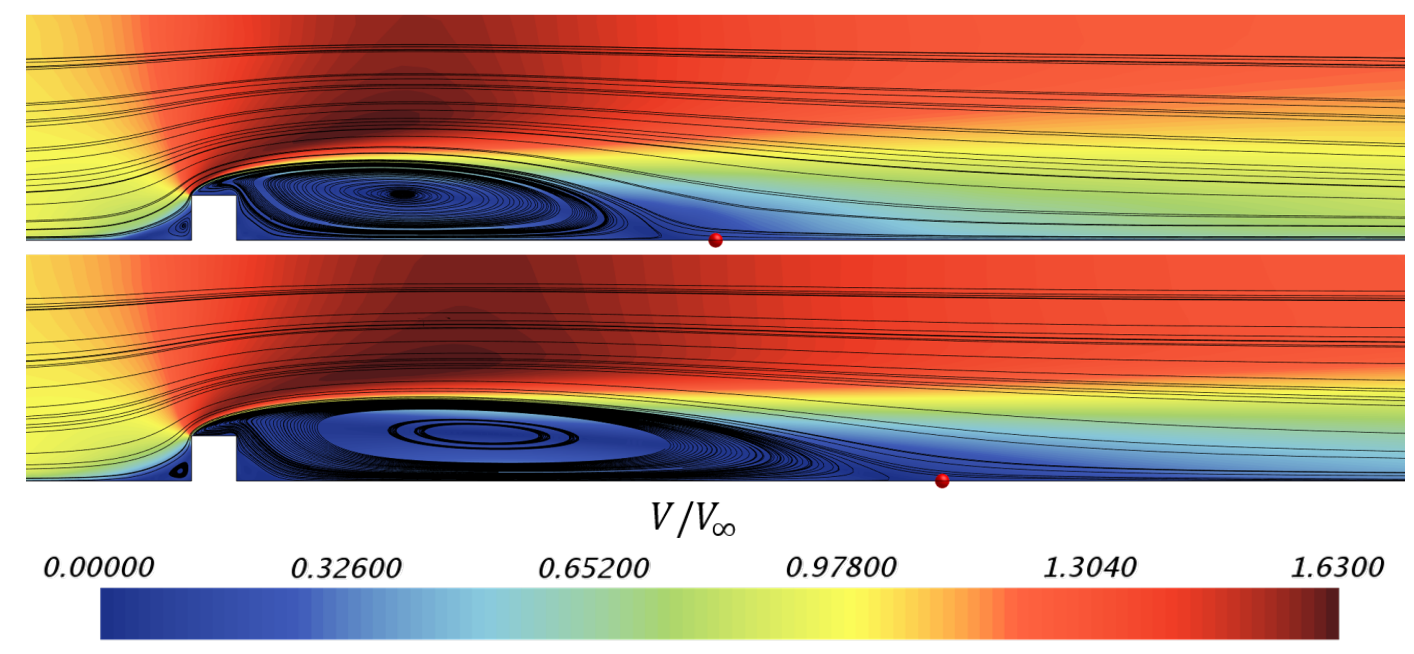

Figure 8: Time averaged velocity using LES (top) and RANS (bottom) for the mesh with $N_{\text {elements }} \approx 20 \times 10^{6}$

From the computational calculations, the reattachment location can be identified as the point where the time averaged wall shear stress is in the same direction as the main flow. This criteria is similar to the one stated for the PIV measurements when the velocity is measured close enough to the wall. This fact can be easily observed in Figure 9, where the non dimensional $x$ component of the wall shear stress $\left(\frac{\tau_{x}}{\frac{1}{2} \rho_{\infty} V_{\infty}^{2}}\right)$ is shown for RANS and LES computations. While the LES results are shown for both the finer and coarser meshes, the RANS is only shown for the fine one in order to ease the interpretation of the Figure. Notice that, downstream of the step, both RANS and LES calculations predict the same value for the wall shear stress for non-dimensional distances below $x / L_{r e f} \approx 5$. Nevertheless, the LES scheme predicts that the wall shear stress reaches zero at a shorter distance, thus leading to a smaller recirculation bubble. The value of the location of the reattachment length is shown at Table 3 for the different grids for LES and RANS. Note how the coarser and finer LES resolutions allows to obtain a similar value for this parameter.

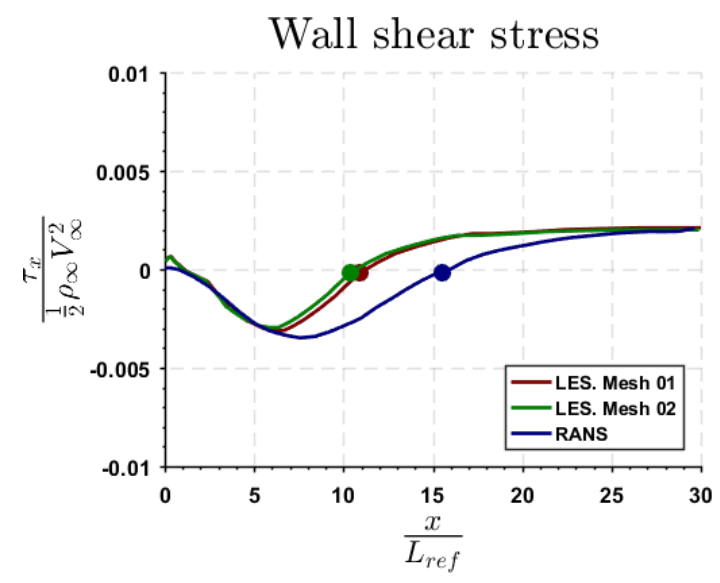

Figure 9: Average non dimensional wall shear stress after the step (left) and over the step (right) $N_{\text {elements }} \approx 20 \times 10^{6}$ and identification of the reatahcment location 
Table 3: Value of the reatachment mesh for each of the studied computational calculations. Comparison with the experimental value

\begin{tabular}{|c|c|c|c|c|c|}
\cline { 2 - 6 } \multicolumn{1}{c|}{} & LES. Mesh 01 & RANS. Mesh 01 & LES. Mesh 02 & RANS. Mesh 02 & $\exp$ \\
\hline \hline$\left(x_{\text {reattach }} / L_{\text {ref }}\right)$ & 10.9 & 16.8 & 10.7 & 15.7 & $10.0 \pm 0.7$ \\
\hline
\end{tabular}

It can be stated then that the agreement between LES and the experiments is good for this parameter, for both meshes, whereas the RANS computation fails in predicting the reattachment length. A reasonable explanation of why RANS methodology overpredicts the extension of the recirculation zone can be given as follows: as it can be observed in Figure 10, in order to calculate the high values of turbulent kinetic energy existing in the last part of the shear layer, a zone with a very high turbulent viscosity ratio (up to $\mu_{t} / \mu>900$ ) is needed. Such high value of the turbulent viscosity leads to an effective Reynolds number much lower than the real one. It is well known that, for the case of a medium-high Reynolds backstep the reattachment length tends to increase when decreasing the Reynolds number, as it can be found, for instance, in the works of Armaly et al. [42] or Kostas et al. [43]. Similar results were found by Tropea and Gackstatter [44] or Durst et al. [45] for the case of a wall-mounted 2D obstacle, explaining why the RANS solution tends to overpredict this parameter.

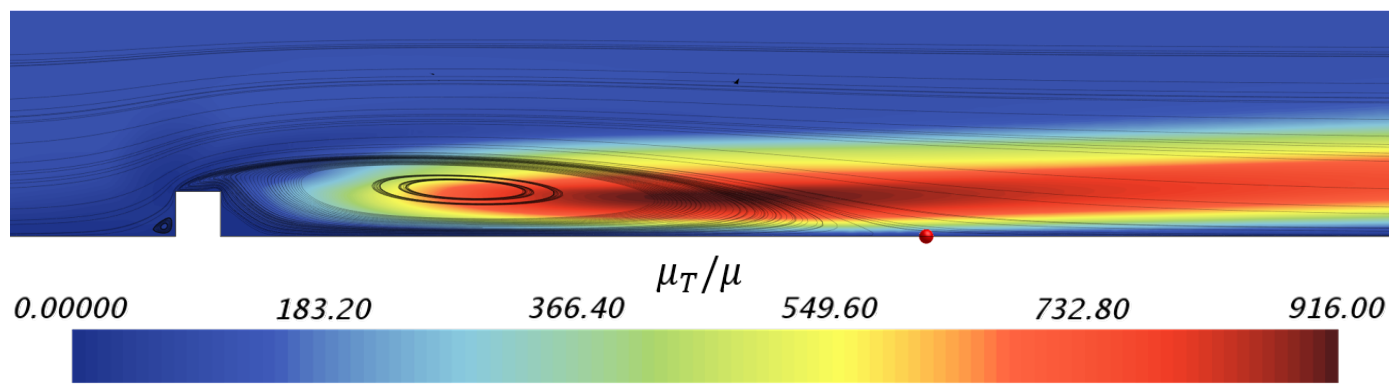

Figure 10: Turbulent viscosity ratio for the RANS computation with the mesh of $N_{\text {elements }} \approx 20 \times 10^{6}$

In order to check the resolution of a LES scheme, Celik et al. [46] proposed an index of quality that has been successfully tested on the works of Lucius and Brenner [47], Konnigk et al. [48] or Dastbelaraki et al. [49]. Another useful indicator derived from these works, which can be used for almost any complex flow, relates the ratio of the turbulent kinetic energy of the calculated non-filtered structures $\left(k_{c}\right)$ and the kinetic energy introduced by the subgridscale $\left(k_{S G S}\right)$, as defined in Equation 10 ([21],[28]):

$$
\eta=\frac{k_{c}}{k_{T O T A L}}=\frac{k_{c}}{k_{c}+k_{S G S}}
$$

When $\eta>0.7-0.8$ the energy content of the turbulent structures is correctly resolved $(\eta=1$ means DNS resolution). Both the resolved and the modeled turbulent kinetic energy can be calculated as defined in Equations 11 and 12:

$$
\begin{gathered}
k_{c}=\frac{1}{2}\left(<v_{x}^{\prime 2}>+<v_{y}^{\prime 2}>+<v_{z}^{\prime 2}>\right) \\
k_{S G S}=C_{t} \frac{\mu_{t}}{\rho} S
\end{gathered}
$$

Here, $v_{i}^{\prime}=v_{i}-<v_{i}>$ represents the deviation of the resolved $i^{\text {th }}$ component of the velocity with respect to its time averaged value; $C_{t}=3.5$ is a constant of the subgrid model; $\mu_{t}$ represents the turbulent viscosity and $\boldsymbol{S}$ is the strain tensor computed with the resolved velocity field.

In this article the level of resolution of the LES computation will be analyzed for the case of the coarser computational grid, with $N_{\text {elements }} \approx 4 \cdot 10^{6}$, as the finer one will provide a higher level of resolution. In Figure 11 the resolved (top), the modeled (middle) and the total (bottom) turbulent kinetic energy are shown at the mid plane. It should be 
noticed that the computed and total kinetic energy are quite similar over the whole domain. The most important source of modeled subgrid kinetic energy can be found at the shear layer just over the step, where a significant flow velocity gradient exists.
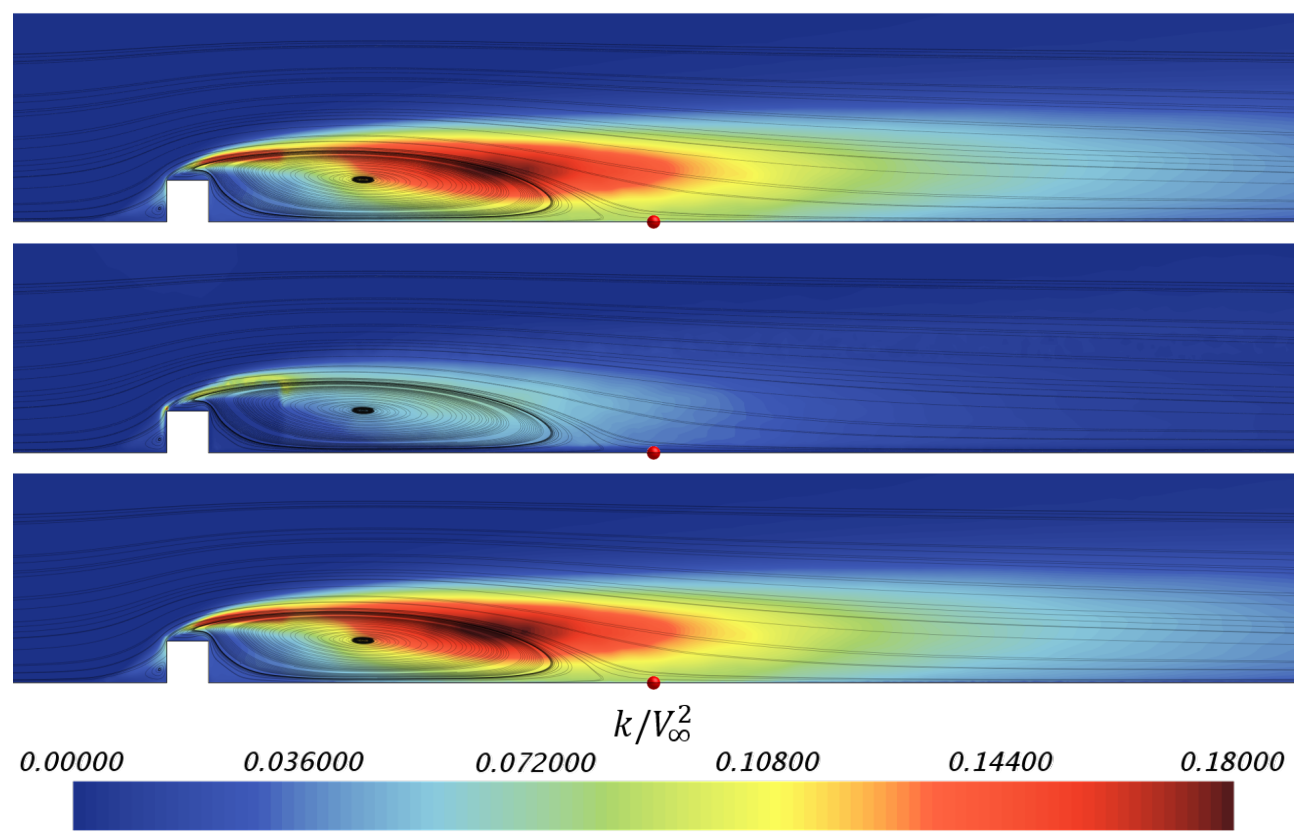

Figure 11: Resolved (top), subgrid scale (middle) and total turbulent kinetic energy at the midplane. Computation for the mesh with $N_{\text {elements }} \approx$ $4 \times 10^{6}$

In order to check the level of resolution of the current simulation, Figure 12 shows the ratio of resolved to total turbulent kinetic energy. Observe that, downstream of the step, it is possible to find a value of $\eta \geq 0.7$ for the whole domain. Upstream, where the mesh is coarser, a very low resolution zone is found. Nevertheless, as, in agreement with Figure 11, the turbulence kinetic energy is quite low, and the flow just downstream of the step is highly dominated by the detached flow, the resolution was considered sufficient for the present study. Moreover, it should be noted that refining the upstream zone would lead to a significant increase in the computational cost with small added value. Figure 13 shows the percentile distribution of the ratio of turbulent kinetic energy (left) and wall $y^{+}$for the whole domain. Notice that $y^{+} \leq 1$ for $98 \%$ of near-wall cells and $\eta \geq 0.80$ for $92 \%$ of the cells.

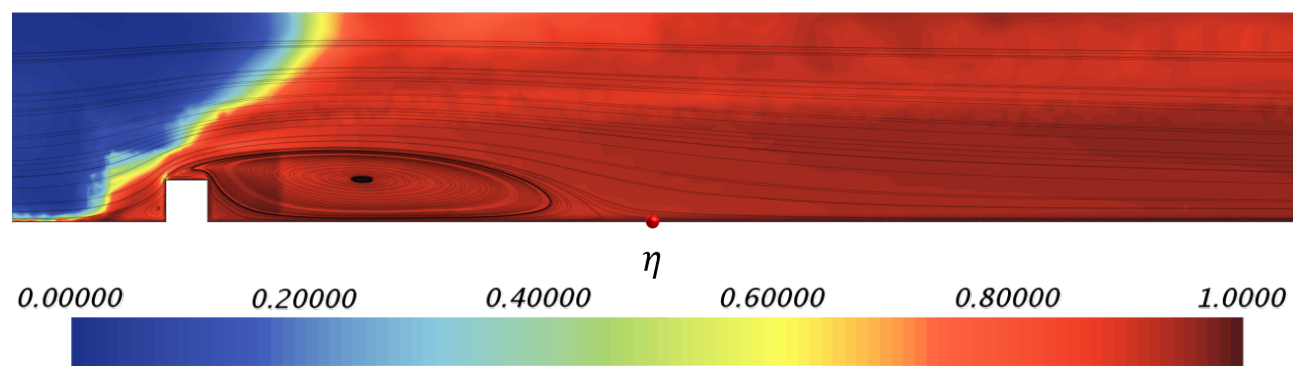

Figure 12: Ratio of resolved over total turbulent kinetic energy at the midplane. Computation for the mesh with $N_{\text {elements }} \approx 4 \times 10^{6}$ 

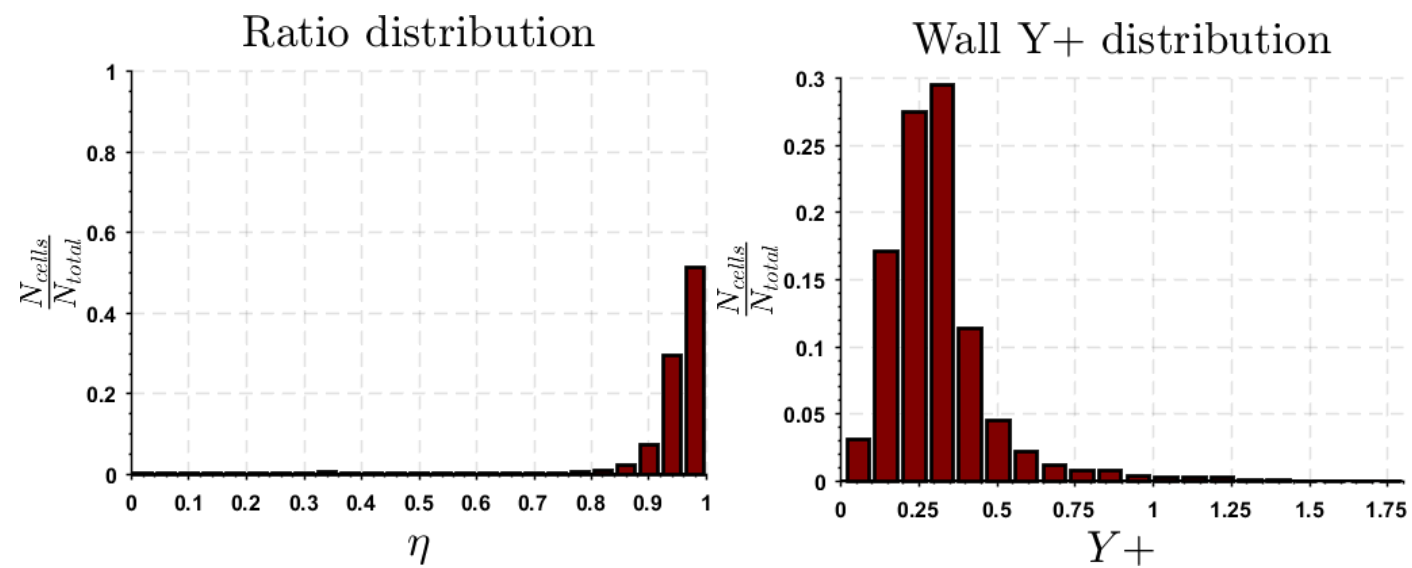

Figure 13: Distribution of the ratio of resolved over total turbulent kinetic energy and wall $y^{+}$. Computation for the mesh with $N_{\text {elements }} \approx 4 \times 10^{6}$

The unsteady velocity field was constantly recorded in some points of interest. As an example, Figure 14 shows the analysis of the deviation of the velocity components from their mean value at a point located over the shear layer, at a point $P_{1}$, located at the centerline, and defined by $x / L_{r e f}=20 / 3$ and $y / L_{r e f}=4 / 3$, for the mesh with $N_{\text {elements }} \approx 20 \times 10^{6}$. The left plot shows the time history of the velocity while the right one shows its frequency content. Again the absence of any kind of dominant frequency can be observed.

This is corroborated by the instantaneous velocity field shown in Figure 15 for an arbitrary time step at the mean plane and at the wake. In this Figure, it can be observed how the largest turbulent structures in the field are approximately of the same size as the obstacle. Also it should be noted how it is not possible to identify any kind of coherent vortex shedding, which is in agreement with the non-existence of dominant frequencies.
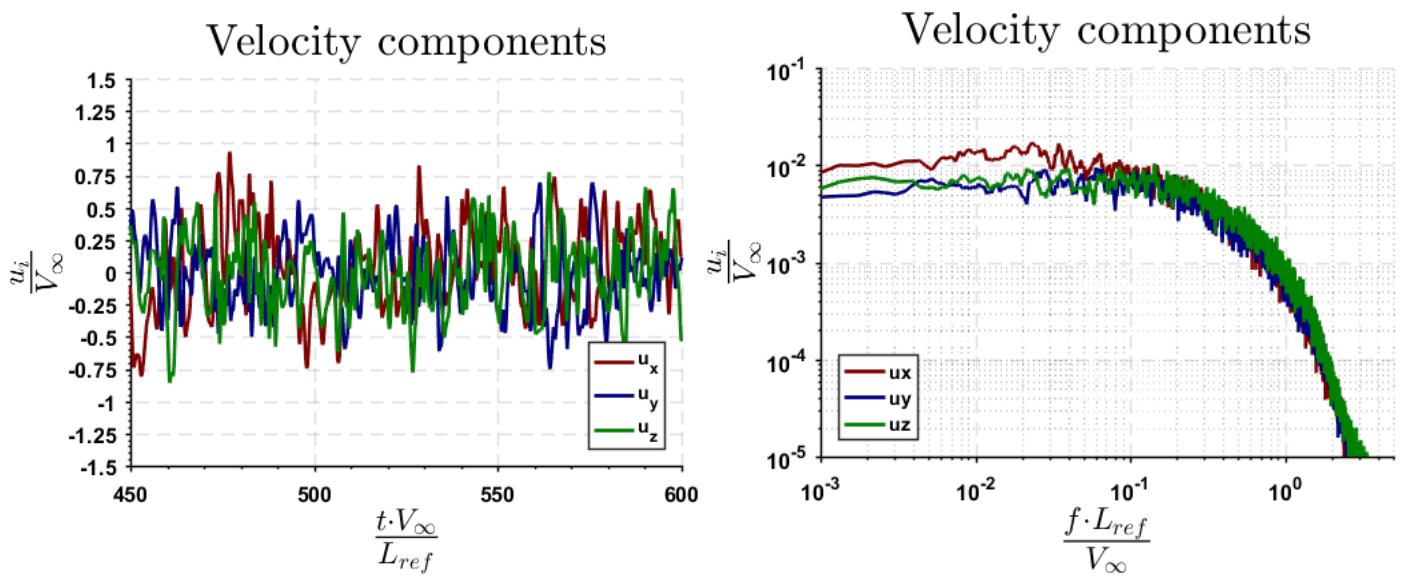

Figure 14: Velocity time history (left) and frequency content (right) at a point $P 1$, located near to the main shear layer. Computations for the mesh with $N_{\text {elements }} \approx 20 \times 10^{6}$ 


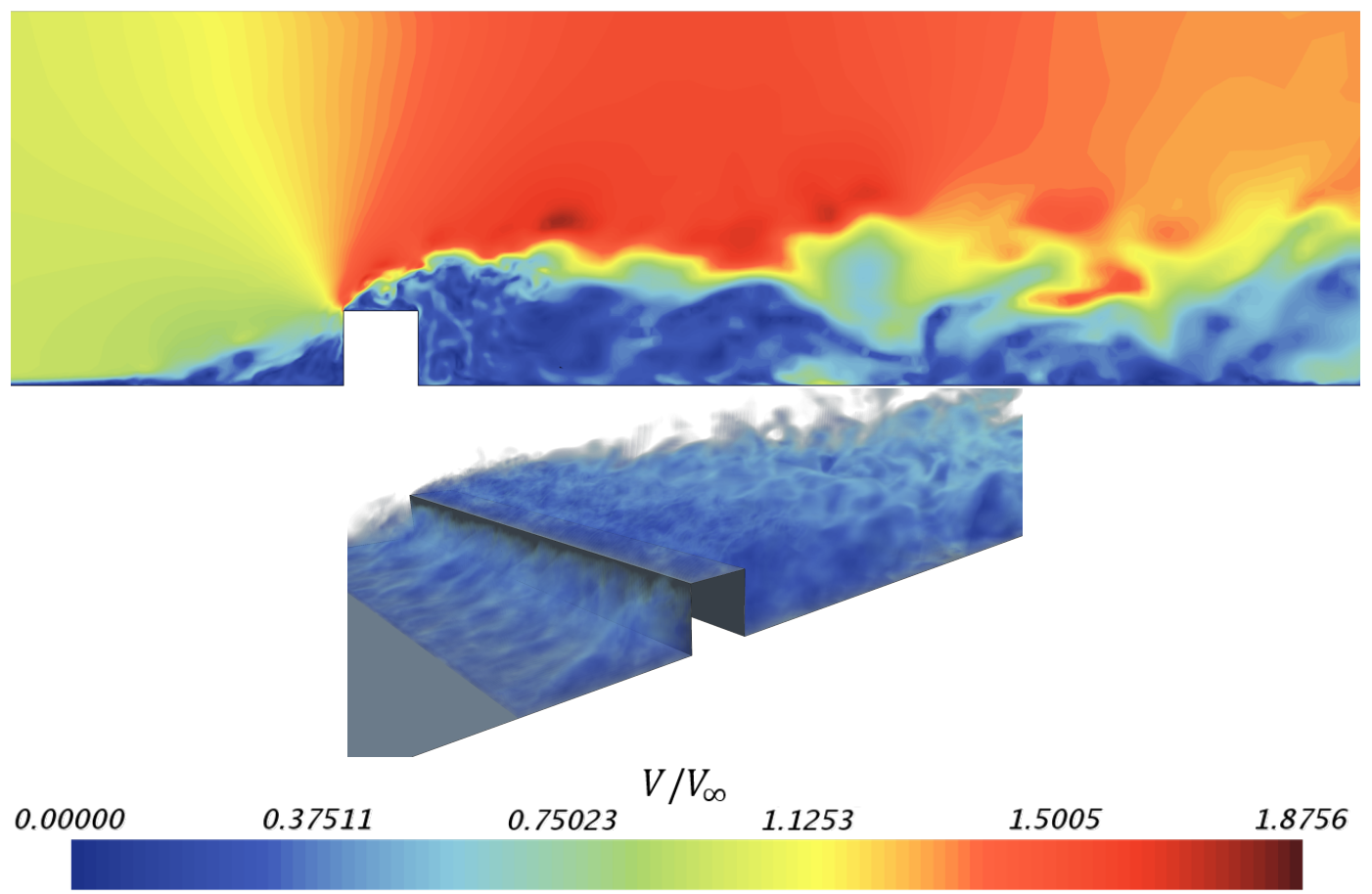

Figure 15: Visualization of turbulent strucures at an arbitrary instant for the computation with $N_{\text {elements }} \approx 20 \times 10^{6}$. Contour of instantaneous velocity field at the midplane (top) and volumetric render of the low velocity at the wake (bottom)

The spectrum of the kinetic energy content at point P1 is shown at Figure 16 (left) for the two different meshes, it should be noted how the refined mesh allows one to obtain a meaningful higher level of energy. The direct effect of this will be later explained when examining the prediction of the flexible plate vibration. Note how the inertial subrange can be identified by the $-5 / 3$ slope, ranging from $S t=0.35$ up to $S t=1.681$. For the large scale energy-containing subrange, the energy spectrum is quite flat, which agrees with the absence of any kind of dominant frequency. A similar trend can be observed at Figure 16 (right), where the frequency content of the pressure coefficient at a the point G5, located at the plate, is shown. Note, how, in a similar way as for the energy spectra, higher values of the frequency content are observed for the finer mesh.
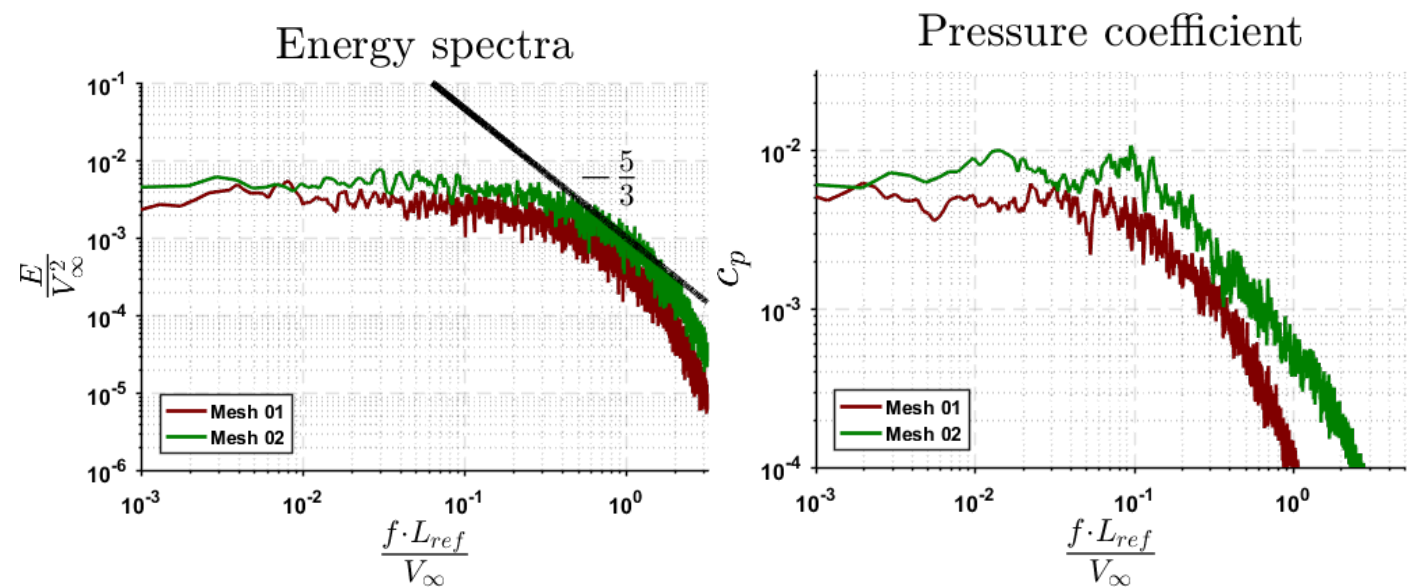

Figure 16: Energy spectra at the point located near to the shear layer, $P 1$ (left) and frequency content of the pressure coefficient at a point located over the plate, at point 65 . Comparison between different meshes 


\subsection{Structural model validation}

As it could be expected, the prediction of the flow-induced vibration is highly influenced by the quality of the structural model. Moreover, when the plate is excited by a turbulent unsteady pressure, with a significant spectral content in a wide frequency range, a correct estimation of the natural frequencies of the system becomes vital.

Although it could be argued that, due both to the pre-load state and the added mass effect, the structural eigenfrequencies will not be the same as those corresponding to the structure response under the action of a moving fluid, in fact significant differences should not be expected, due to the low values of the mean pressure, fluid density and Mach number. Therefore the isolated structure model is a good approximation, as verified by the study of Frampton [50].

In Table 4 the numerical values of the first 10 eigenfrequencies are shown. Notice that, although they correspond to the vibration of the plate in vасио, they have been non-dimensionalized by using the fluid flow inlet velocity $\left(f^{*}=f \cdot L_{r e f} / V_{\infty}\right)$. The parameters for non-dimensionalization were chosen in order to maintain the coherence on the presentation of the results of the current work. From Table 4 it can be seen how, despite the simple set of boundary conditions which was supposed for the current study, the agreement between prediction and measurement is good.

Table 4: Structural eigenfrequencies of the flat plate

\begin{tabular}{|l|c|c|c|c|c|c|c|c|c|c|}
\cline { 2 - 12 } \multicolumn{1}{c|}{} & $f_{1}^{*}$ & $f_{2}^{*}$ & $f_{3}^{*}$ & $f_{4}^{*}$ & $f_{5}^{*}$ & $f_{6}^{*}$ & $f_{7}^{*}$ & $f_{8}^{*}$ & $f_{9}^{*}$ & $f_{10}^{*}$ \\
\hline \hline Num & 0.104 & 0.143 & 0.237 & 0.243 & 0.292 & 0.376 & 0.382 & 0.461 & 0.513 & 0.514 \\
\hline Exp & 0.112 & 0.140 & 0.227 & 0.249 & 0.295 & 0.359 & 0.389 & 0.425 & 0.484 & 0.527 \\
\hline$\varepsilon$ & 0.036 & 0.024 & 0.043 & -0.025 & -0.009 & 0.071 & 0.020 & 0.078 & 0.056 & 0.025 \\
\hline
\end{tabular}

Mode 1

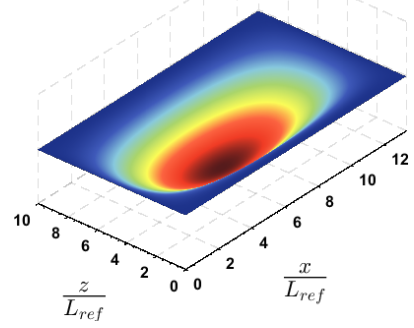

Mode 4

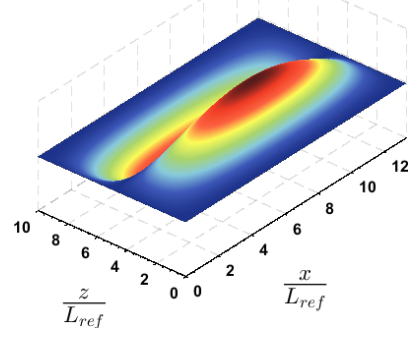

Mode 2

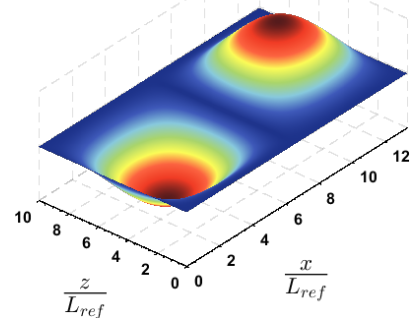

Mode 5

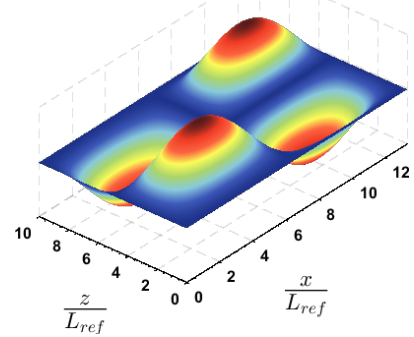

Mode 3

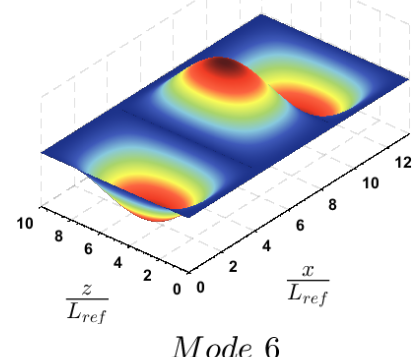

Mode 6

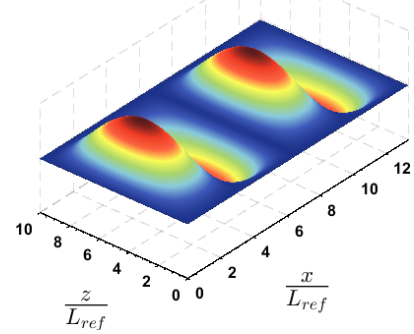

Figure 17: Calculated modal displacements

To validate the prediction of the modal shapes, the numerical $\left(\phi_{i}^{\text {num }}\right)$ and experimental $\left(\phi_{i}^{\text {exp }}\right)$ eigenvectors are compared by means of the Modal Assurance Criteria (MAC) [51] which allows to define a MAC matrix, as stated by Equation 13:

$$
M A C_{i j}=\left(\frac{\phi_{i}^{\text {num }}{ }^{T} \cdot \phi_{j}^{\text {exp }}}{\left\|\phi_{i}^{\text {num }}\right\|\left\|\phi_{i}^{\text {exp }}\right\|}\right)^{2}
$$


where, $M A C_{i i}=1$ means perfect agreement between the numerical and experimental mode shape and $M A C_{i j}=0$ means perfect orthogonality between the numerical $i^{\text {th }}$ and the experimental $j^{\text {th }}$ eigenfunctions. Figure 18 shows a visual representation of this matrix, where a good agreement between the experimental and numerical data can be observed. MAC coefficients are always above 0.70 at the diagonal and below 0.20 for the terms out of the diagonal. However, it can be noted how modes $4^{\text {th }}$ and $3^{\text {rd }}$ are slightly superimposed. This difference can be attributed to the closeness of the frequencies of these modes (there exist a difference of less than a $3 \%$ both in measurements and computations).

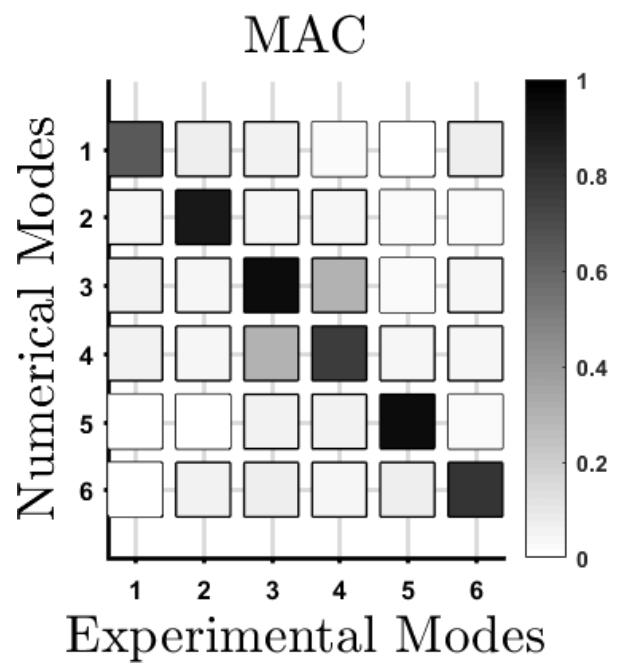

Figure 18: Modal Assurance Criterion matrix

\subsection{Flow induced vibrations}

The detached flow downstream of the step generates a turbulent fluctuating pressure, which excites the back plate. As a consequence, this structure experiences a time averaged mean displacement due to the action of the time average pressure and a fluctuating displacement due to pressure fluctuations. Therefore, the correct estimation of the pressure acting over the plate becomes of crucial importance for the correct estimation of the displacement. Figure 19 (left) shows the evolution of the time-averaged pressure coefficient downstream, comparing the RANS and LES results, computed at the mean line which was shown at Figure 2. Figure 19 (right) shows the mean displacement appearing in response to such mean pressure.
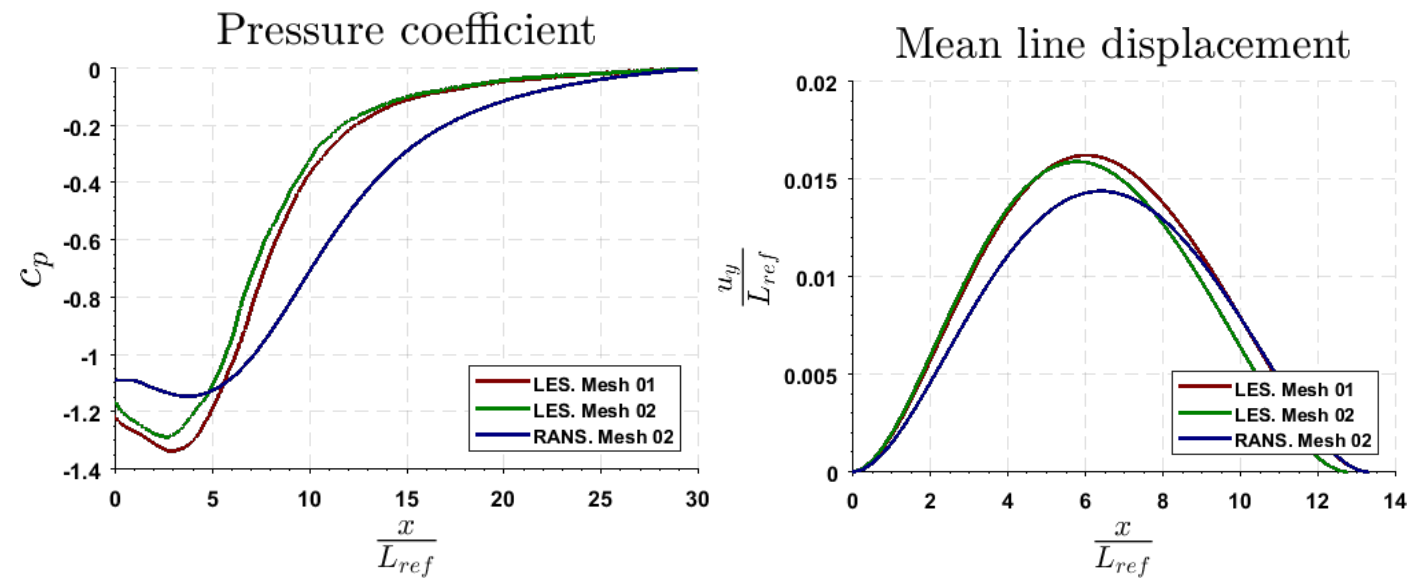

Figure 19: Time averaged pressure coefficient (left) and mean line plate displacement (right) after the step. Comparison between LES and RANS computations using different meshes 
As it can be observed, the LES calculation predicts a lower value of the pressure in the recirculation bubble. Also, the extension of the low pressure zone is less pronounced and the minimum value is located a $30 \%$ closer to the obstacle position. These results are highly in agreement with the best capacity of the LES computation to accurately predict the extension of the recirculation zone, as could be deduced from literature comparing the works of Yang and J.H. [19] or Werner and Wengle [52], where the flow over a similar configuration is analyzed using LES and the works of Schmidt and Thiele [53] or Ariff et al. [54], who used RANS in order to characterize the flow. Note how, As the location of the lowest value of the pressure is not in the center of the plate, the deformation which it experiences is not symmetric.

Table 5 shows a comparison of the main values extracted from Figure 19 which are: (i) the location of the point of minimum pressure coefficient, $\left(\frac{x}{L_{r e f}}\right)_{c_{p_{m}}}$; (ii) the value of the minimum pressure coefficient, $c_{p_{\min }}$; (iii) the location of the maximum plate displacement $\left(\frac{x}{L_{r e f}}\right)_{u_{\text {max }}}$ and (iv) the value of the maximum plate displacement $\frac{u_{y_{\max }}}{L_{r e f}}$. Note how, despite the location of the minimum pressure coefficient point is highly overpredicted by the RANS calculation by a $30 \%$ in comparison with LES this translates at a difference of only $11 \%$ on the prediction of the location of the point of maximum amplitude.

Table 5: Time averaged displacement predictions using different turbulence modelling and computational grids

\begin{tabular}{|l|c|c|c|c|}
\cline { 2 - 5 } & $\left(\frac{x}{L_{r e f}}\right)_{c_{p_{\min }}}$ & $c_{p_{\min }}$ & $\left(\frac{x}{L_{\text {ref }}}\right)_{u_{\text {ymax }}}$ & $\frac{u_{\text {ymax }}}{L_{\text {ref }}}$ \\
\hline \hline LES. Mesh 01 & 2.91 & -1.34 & 5.99 & 0.0162 \\
\hline RANS. Mesh 01 & 4.07 & -1.47 & 6.67 & 0.0149 \\
\hline LES. Mesh 02 & 2.75 & -1.30 & 5.75 & 0.0159 \\
\hline RANS. Mesh 02 & 3.99 & -1.44 & 6.53 & 0.0144 \\
\hline
\end{tabular}

Figure 20 shows the spatial distribution of the time-averaged non-dimensional displacement of the plate for the LES (top) and RANS (bottom) models. The point of maximum time-averaged displacement is highlighted in both figures. It can be observed that, as expected, the displacement field is symmetric with respect to $\mathrm{z}$ axis and the maximum displacement point is displaced towards the step. This fact is more clearly noticeable for the LES calculation. 


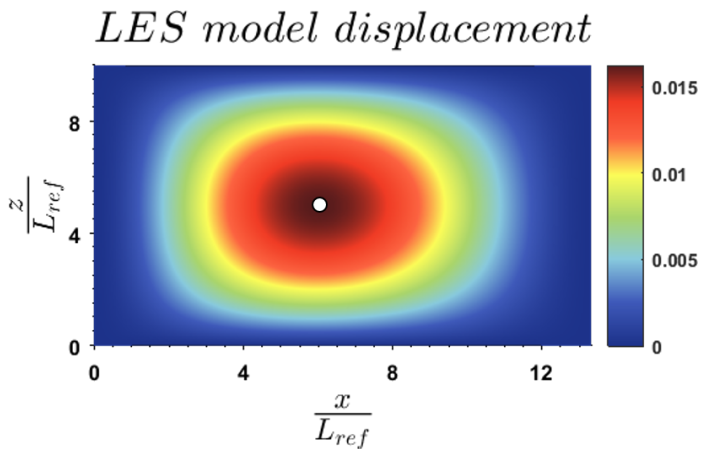

\section{RANS model displacement}

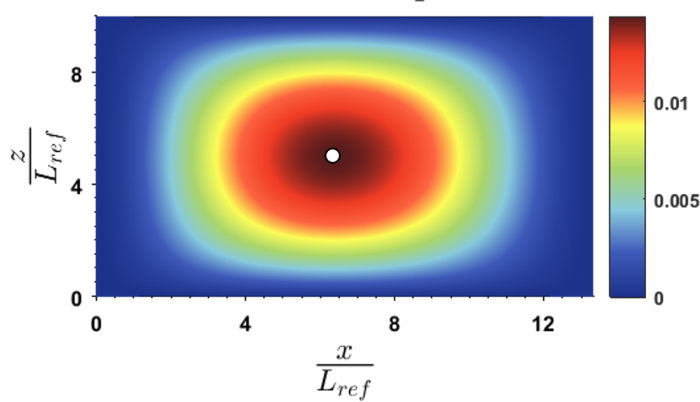

Figure 20: Time averaged displacement field with LES (top) and RANS (bottom) computations. Computation with the mesh of $N_{\text {elements }} \approx 20 \times 10^{6}$

The transient flow field which features have been analyzed so far, induces a fluctuating pressure field over the plate located at the rear part of the step. This fluctuating pressure excites the plate, which acquires a vibrational motion superimposed onto the mean displacement analyzed in Figure 19.

Figure 21 shows the behavior of the fluctuating pressure at four points on the plate: point G1, located at $x / L_{\text {ref }}=1$; G3 at $x / L_{r e f}=3$; G5 at $x / L_{r e f}=5$ and G8 at $x / L_{r e f}=8$. All the points were located at the middle plane $\left(z / L_{r e f}=5\right)$. The frequency content at low frequencies is in agreement with the averaged pressures shown in Figure 19, tending to lower values (closer to atmospheric pressure) as the point is located farther from the obstacle. Also, from $S t>0.15$ the frequency content of the pressure for all the shown points tends to rapidly decay.
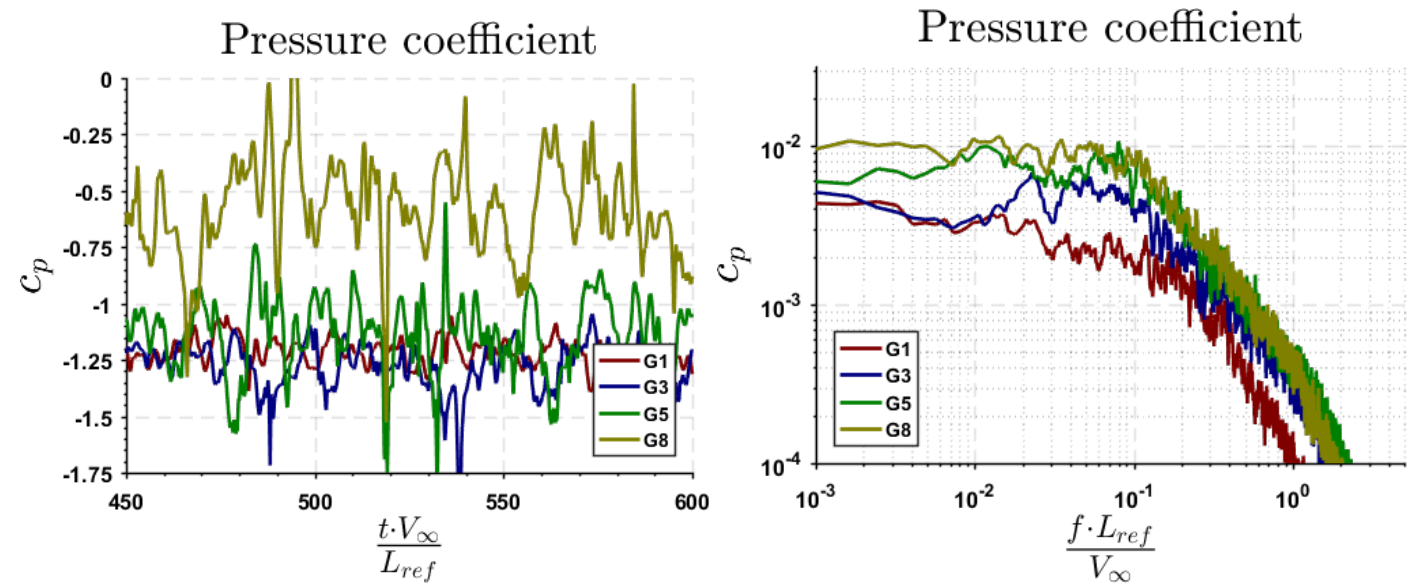

Figure 21: Time history (left) and frequency content (right) of the unsteady pressure coefficient at points located over the plate for the mesh of $N_{\text {elements }} \approx 20 \times 10^{6}$ 
The fluctuating pressure history was used as an excitation for the flat plate, whose deformations were computed and compared with experimental measurements. Figure 22 shows the displacement field for six different frequencies close to the eigenfrequencies of the structural system. A visual comparison with Figure 17 suggest all the modes participate in the deformation of the plate at the evaluated frequency range. The modal participation of the first modes was quantitatively calculated for these frequencies in a similar way as proposed by Chopra [55]: the response of the structure to a vibratory loading can be expressed in accordance with Equation 14, assuming a system with $N$ participating modal forms:

$$
u(x, z, f)=\sum_{i=1}^{N} \eta_{i}(f) \phi_{i}(x, z)
$$

where $\phi_{i}$ denotes the $i^{\text {th }}$ eigenfunction, which was previously calculated and shown at Figure 17 and $\eta_{i}$ represents contribution of the $i^{\text {th }}$ mode to the response of the plate at frequency $f$. Figure 23 shows these values in order to specify the contribution of the first seven modes to the total response. For an easier interpretation, they have been scaled so that a value of 1 correspond to the maximum modal contribution of the first mode for the first frequency.

Note how the frequencies of the $3 \mathrm{rd}$ and 4 th modes are very close (less than $3 \%$ of separation). Also, it can be easily observed that, at the frequency of the fourth mode ( $\mathrm{St} \approx 0.243$ ), the contributions of the 3rd and the 4th modes are comparable, thus supporting the explanation given of why the 4th mode cannot be clearly observed in the frequency response of the averaged surface displacement, as will be stated later.
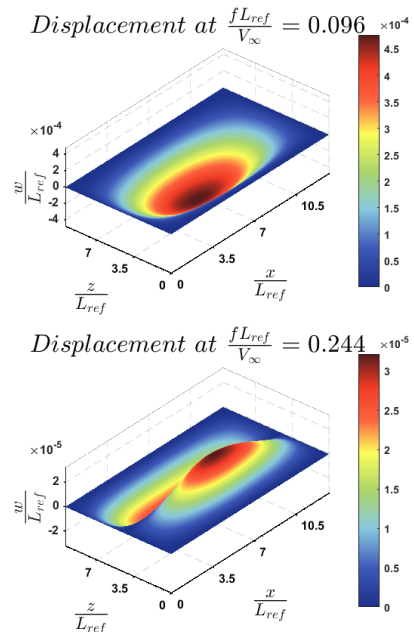

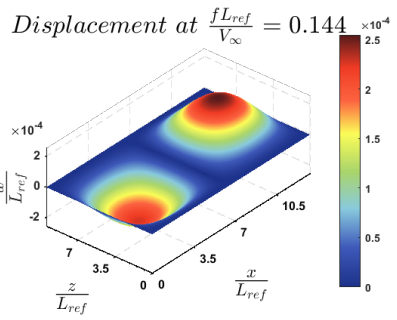

Displacement at $\frac{f L_{r e f}}{V_{\infty}}=0.293_{3^{10^{-5}}}$

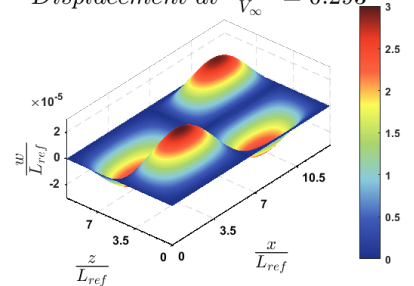

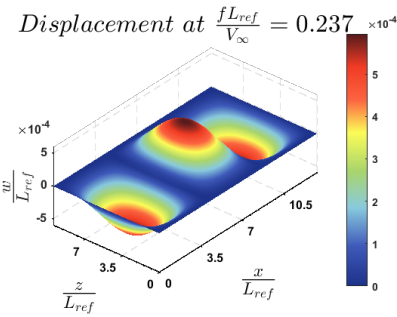

Displacement at $\frac{f L_{\text {ref }}}{V_{\infty}}=0.362 \times 10^{-6}$

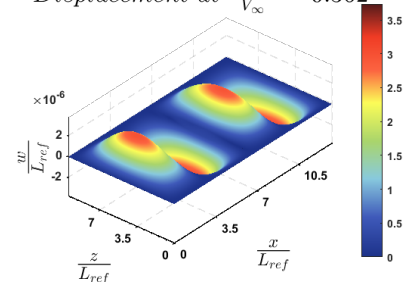

Figure 22: Frequency response of the plate displacement under the action of the turbulent pressure field at different frequencies 


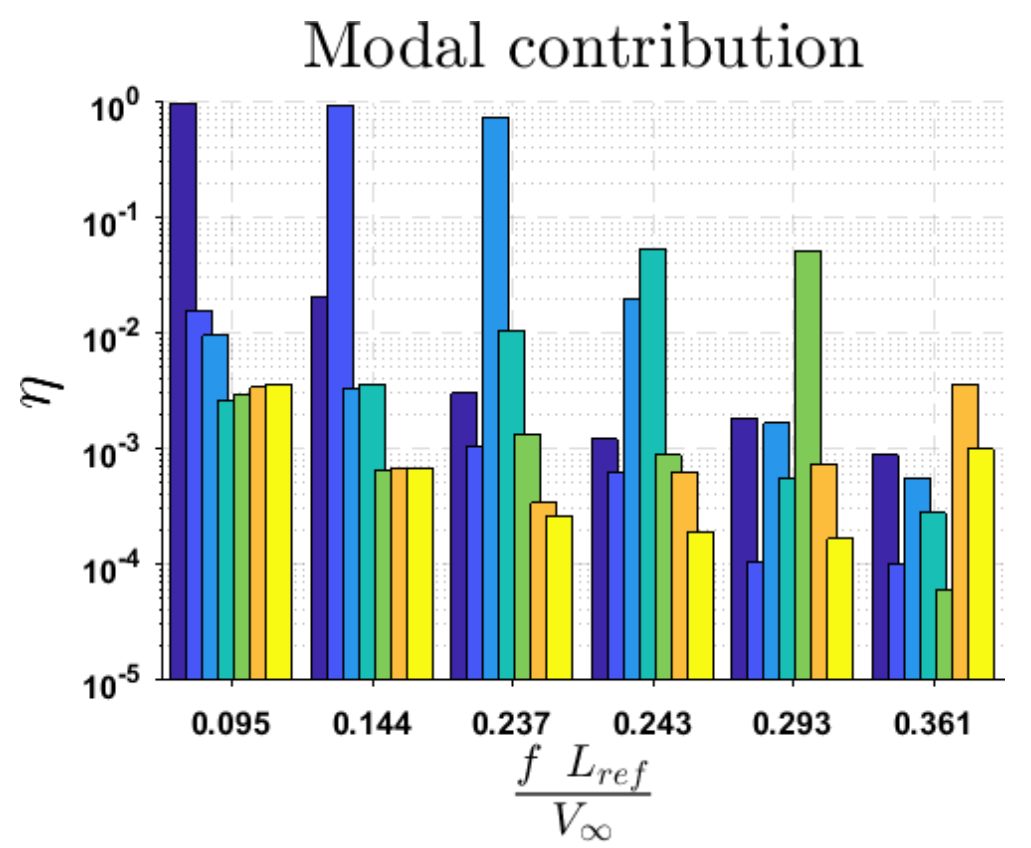

Figure 23: Modal contribution of each eigenvector to the total displacement of the plate under the action of the turbulent pressure field at different frequencies. Only the first 7 modes are shown

Additionally, Figure 24 (left) shows the spatial-average frequency response of the displacements over the plate, which are calculated as stated in Equation 15.

$$
\bar{u}_{y}(f)=\sqrt{\frac{\iint_{\text {plate }} u_{y}^{2}(f) d A}{A_{\text {plate }}}}
$$

Figure 24 (left) shows a comparison between the experimental and computed spatial-averaged vibration of the plate for a range of frequencies ranging from $f \cdot L_{r e f} / V_{\infty} \approx 0$ to $f \cdot L_{r e f} / V_{\infty} \approx 1.300(f=1900 \mathrm{~Hz})$. Note how, for frequencies below $f \cdot L_{r e f} / V_{\infty} \approx 0.800$, both the coarse and the fine mesh provide results which are excellent in agreement with those deduced from the experiments. For frequencies above this value it is shown that the computations with the coarse mesh tend to underpredict the level of the displacement although, as expected, exhibiting the peaks at the same frequencies than the other computation.

As opposed to the pressure spectrum, some peaks can be observed in the displacement spectrum. The plate resonates at all its eigenfrequencies, which can be visually identified from Figure 24 and compared with the eigenvectors shown in Figure 17. Only the 4th structural mode is hardly observed in the figure, but as it is very close to that of the third mode it may be masked in the frequency response. The discrepancy between computations for medium values of the frequency can be explained due to the filtering effect which the gross mesh exhibits over the energy content.

In order to compare with previous works, Figure 24 (right) shows the results which were obtained by Schafer et al. [14]. Note how, as the plate characteristics are different, current results can only be qualitatively compared with this. Nevertheless, it is important to note how the current computation provides more accurate results both in excitation level and peaks location.

There are, however, some discrepancies between the current calculation and the experiments. For example, a discrepancy of an $8 \%$ was found on the prediction of the first eigenfrequency. This can mainly due to the approximations made on the boundary conditions. Using non-infinitely stiff boundary conditions could provide a better prediction, but it is out of the scope of this contribution. The same reasoning could be argued in order to explain why there are some discrepancies at the peaks location at high frequencies. 

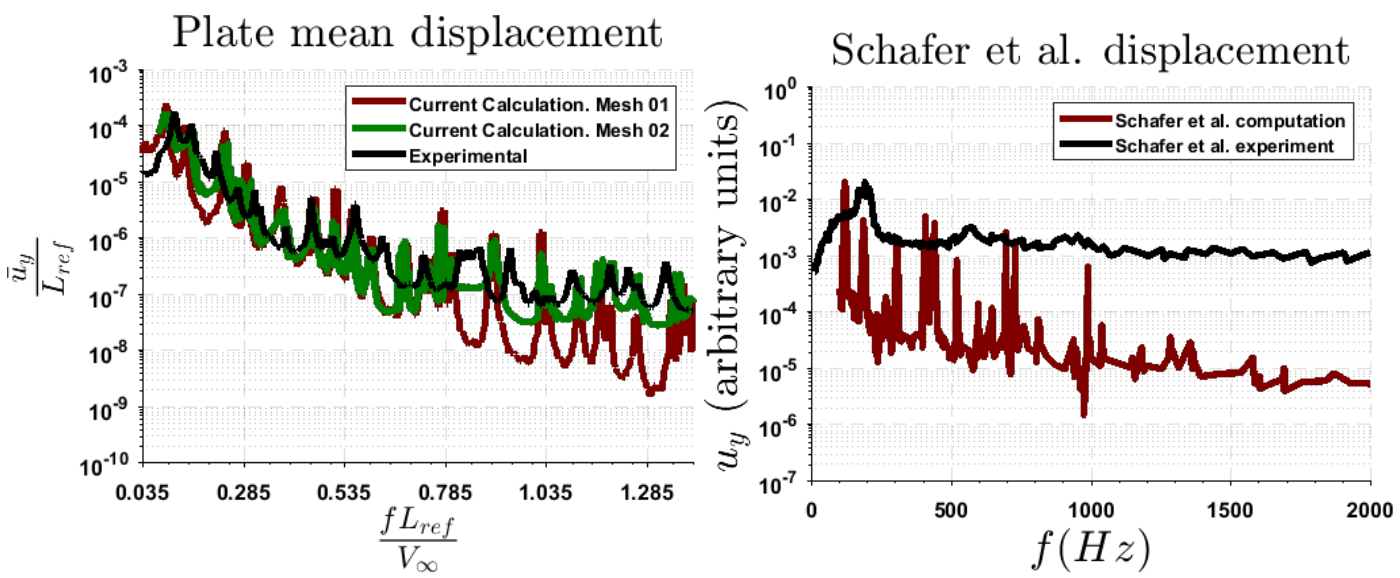

Figure 24: Mean displacement spectra for the current calculation (left) and collection of the displacements results taken from Schafer et al. for a plate of thickness $h=40 \mu \mathrm{m}$ (right)

\section{Conclusions}

During the current work, numerical and experimental investigations on the flow-induced vibration of a flat plate have been presented. In particular, the vibration of a plate excited by the turbulence generated by an upstream wall mounted obstacle have been analyzed.

The main hypothesis taken for this work is that the interaction between the fluid flow and the flexible plate can be considered to be coupled in only one direction, i.e., the fluid flow is responsible of exciting the vibrational response of the flexible plate but those vibrations should not significantly affect the fluid field itself. This hypothesis is supported by:

- The high value of the non dimensional stiffness, $\frac{E}{6\left(1-v^{2}\right) \rho_{\infty} V_{\infty}^{2}}\left(\frac{h}{L_{r e f}}\right)^{3}$.

- The low value of both the time average and frequency content of the computed displacements, which indicate that the fluid domain shape should not be significantly modified by the displacement of the plate.

- The agreement between the computations and the vibrational measurements.

About the computation of the time average displacement of the plate, RANS fluid flow modeling has been shown to provide similar results as LES, indicating that the former could be used for similar cases for preliminary predictions, even considering that RANS tends to considerably over-predict the reattachment length.

Additionally, the influence of grid refinement on the LES results has been explored: it has been observed how a relatively coarse mesh can be used for obtaining a reasonable vibrational response at low frequencies and qualitatively results for medium to high values of the frequency. This allows to conclude that, for the pre-design phase of systems working under similar working conditions (namely, similar values of stiffness and fluid flow velocity and density) a coarse mesh can be used for obtaining valuable results in industrial time scales. When the numerical grid is refined it is possible to achieve better agreement between computation and experiments for higher values of the frequency while for low values of this parameter, the results basically remain unchanged.

The current work can serve as a guideline for the CFD prediction of turbulent excited structures, allowing to infer which are the most important parameters when facing with these problems:

- For the usual values of velocity and stiffness found in the automobile industry, the assumption of one way coupling is usually justified.

- A coarse mesh allows obtaining reasonably good results in relatively short times, which can be later improved, if needed, by a grid refinement. 
- It is important to generate an accurate structural eigenfrequency model, as the excitation of the natural modes is the main mechanisms giving rise the flow induced vibrations on high stiffness structures.

- The unsteady flow excitation is mainly governed by the large turbulent structures generated by the wall-mounted obstacle, and thus it is important to obtain a fluid model which is able to provide good resolution of this part of the domain.

Finally, the confirmation of the one-way coupling hypothesis has important consequences. Due to this, the fluid and solid domains can be studied separately. Due to the necessity of solving the turbulent flow fluctuations the first domain will be the most CPU time consumer, but it will have to be solved only once. Having this fluid domain resolution, it will be possible to study later the structural response in a very time-efficient way in a parametric fashion: for instance, changing the plate thickness, material, density... Moreover, the non dimensional expression of the results should allow to extrapolate (at least qualitatively) to different values of the fluid flow velocity.

\section{Acknowledgments}

This work has been partially supported by Universitat Politècnica de València through the grant Programa de apoyo a la Carrera Académica del Profesorado 2018/03/14 and by the Spanish Ministerio de Economía y Competitividad through Grant No. DPI2015-70464-R. The computational resources and services used in this work were provided by the VSC (Flemish Supercomputer Center), funded by the Research Foundation - Flanders (FWO) and the Flemish Government department EWI. The Research Fund KU Leuven and the Flanders Innovation and Entrepreneurship Agency, within the SILENCEVENT project, are gratefully acknowledged for they support.

\section{References}

[1] F. Hilderbrand, E. Reissner, The influence of the aerodynamic span effect on the magnitude of the torsional-divergence velociy and on the shape of the corresponding deflection mode, Tech. Rep. NACA-TN-926, NACA, Massachusetts, Inst of Tech Cambridge, 1944.

[2] R. L. Bisplinghoff, H. Ashley, R. L. Halfman, Aeroelasticity, Courier Corporation, 1996.

[3] U. Jeong, S. Kwon, Sequential numerical procedures for predicting flutter velocity of bridge sections, Journal of Wind Engineering and Industrial Aerodynamics 91 (2003) 291-305.

[4] G. Schewe, A. Larsen, Reynolds number effects in the flow around a bluff bridge deck cross section, Journal of Wind Engineering and Industrial Aerodynamics 74 (1998) 829-838.

[5] B. Augier, P. Bot, F. Hauville, M. Durand, Experimental validation of unsteady models for fluid structure interaction: Application to yacht sails and rigs, Journal of Wind Engineering and Industrial Aerodynamics 101 (2012) 53-66.

[6] Y. Zhang, W. G. Habashi, R. Khurram, Predicting wind-induced vibrations of hight-rise buildings using unsteady CFD and modal analysis, Journal of Wind Engineering and Industrial Aerodynamics 136 (2015) 165-179.

[7] M. Ricci, L. Patruno, I. Kalkman, S. Miranda, B. Blocken, Towards LES as a design tool: Wind loads assessment on a high-rise building, Journal of Wind Engineering and Industrial Aerodynamics 180 (2018) 1-18.

[8] H. Davies, Sound from turbulent boundary layer excited panels, The Journal of the Acoustical Society of America 49 (1971) $878-889$.

[9] W. Graham, Boundary Layer Induced Noise in Aircraft, Part 1: The Flat Plate Model, The Journal of Sound and Vibration 192 (1996) $101-120$.

[10] M. Howe, Influence of Mean Flow on Boundary Layer Generated Interior Noise, Journal of the Acoustical Society of America 99 (1996) 3401-3411.

[11] K. Frampton, Power Flow in an Aeroelastic PLate Backed by a Revererant Cavity, Journal of the Acoustical Society of America 102 (1997) $1620-1627$

[12] M. Springer, C. Scheit, S. Becker, Fluid-struture-acoustic copling for a flat plate, International Journal of Heat and Fluid Flow 66 (2017) 249-257.

[13] S. Mueller, S. Becker, T. Biermeier, F. Schaefer, J. Grabinger, M. Kaltenbaher, D. Blanchet, Investigation of the Fluid-Structure Interaction and the Radiated Sound of Different Plate Structures Deepending on Various Inflows, 15th AIAA/CEAS Aeroacoustics Conference (30th AIAA Aeroacoustics Conference) (2009) 3390.

[14] F. Schafer, S. Mller, T. Uffinger, S. Becker, J. Grabinger, M. Kaltenbaher, D. Blanchet, Fluid-structure-acoustic interaction of the flow past a thin flexible structure, AIAA journal 48 (2010) 738-748.

[15] A. David, F. Hugyes, N. Dauchez, E. Perrey-Debain, Vibrational response of a rectangular duct of finite length excited by a turbulent internal flow, Journal of Sound and Vibration 422 (2018) 146-160.

[16] S. Timoshenko, S. Woinowsky-Krieger, Theory of plates and shells, 1959.

[17] G. Warburton, The vibration of rectangular plates, Proceedings of the Institution of Mechanical Engineers 168 (1954) $371-384$.

[18] K. Bathe, H. Zhang, Finite element developments for general fluid flows with structural interactions, International Journal for numerical methods in engineering 60 (2004) 213-232. 
[19] K. Yang, F. J.H., Large-Eddy Simulation of Turbulent Obstacle Flow Using a Dynamic Subgrid-Scale Model, AIAA Journal 31 (1993) 1406-1413.

[20] X. Zhengtong, I. Castro, LES and RANS for turbulent flow over arrays of wall-mounted obstacles, Flow Turbulence Combust 76 (2006) 291-312.

[21] F. Nicoud, F. Ducros, Subgrid-Scale Stress Modelling Based on the Square of the Velocity Gradient Tensor, Flow, Turbulence and Combustion 62 (1999) $183-200$.

[22] J. Smagorinsky, General Circulation Experiments with the Primitive Equations: Part I, The Basic Experiment, Monthly Weather Review 91 (1963) 99-164.

[23] C. Moussaed, M. Salvetti, S. Wornom, B. Koobus, A. Dervieux, Simulation of the flow past a circular cylinder in the supercritical regime by blending RANS and variational-multiscale LES models, Journal of Fluids and Structures 47 (2014) 114-123.

[24] G. Malloupas, G. Goldin, Y. Zhang, P. Thakre, N. Krishnamoorthy, R. Rawat, D. Gosman, J. Rogerson, G. Bulat, Investigation of an Industrial Gas Turbine Combustor and Pollutant Formation Using LES, ASME Turbo Expo 2017: Turbomachinery Technical Conference and Exposition .

[25] M. Darwish, F. Moukalled, Normalized variable and space formulation methodology for high-resolution schemes, Numerical Heat Transfer 26 (1994) 79-96.

[26] P. Bearman, T. Morel, Effect of free stream turbulence on the flow around bluff bodies, Progress in aerospace sciences 20 (1983) $97-123$.

[27] Y. Nakamura, S. Ozono, The effects of turbulence on a separated and reattaching flow, Journal of Fluid Mechanics 178 (1987) 477-490.

[28] S. Pope, Turbulent Flows, Cambridge University Press, 2009.

[29] G. Ratnam, S. Vengadesan, Performance of two equation turbulence models for prediction of flow and heat transfer over a wall mounted cube, International Jounral of Heat and Mass Transfer 51 (2008) 2834-2846.

[30] S. Archaya, S. Dutta, T. Myrum, R. Baker, Turbulent flow past a surface-mounted two-dimensional rib, Journal of Fluids engineering 116 (1994) 238-246.

[31] D. Ding, S. Wu, Direct numerical simulation of turbulent flow over backward-facing at high Reynolds numbers, Science China 55 (2012) 3213-3222.

[32] F. Menter, Zonal two-equation $k-\omega$ turbulence model for aerodynamic flows, AIAA, Orlando, Florida 93 (1986) 93-2906.

[33] D. Wilcox, Multiscale model for turbulent flows, Proceedings of the 24th AIAA Aerospace Science Meeting 24 (1986) 1311-1320.

[34] P. A. Ullrich, M. Taylor, Arbitrary-order conservative and consistent remapping and theory of linear maps: Part I, Monthly Weather Review 143 (2015) 2419-2440.

[35] P. A. Ullrich, M. Taylor, Arbitrary-order conservative and consistent remapping and theory of linear maps: Part II, Monthly Weather Review 144 (2016) 1529-1549.

[36] P. Jones, First- and second-order conservative remapping schemes for grids in spherical coordinates, Monthly Weather Review 127 (1999) 2204-2210.

[37] G. Taylor, The spectrum of turbulence, Proceedings of the Royal Society of London (1938) 476-490.

[38] D. Roeck, W. Desmet, Experimental acoustic identification of flow noise sources in expansion chambers, Proceedings of ISMA 2018: International Conference on Noise and Vibration Engineering 1 (2008) 455-470.

[39] W. Ren, G. De Roeck, Structural damage identification using modal data II: test verification, Journal of Structural Engineering 128 (2002) 96-104.

[40] N. Roozen, L. Labelle, M. Rychtarikova, C. Glorieux, Determining radiated sound power of building structures by means of laser Doppler vibrometry, Journal of Sound and Vibration 346 (2015) 81-99.

[41] D. Butscher, C. Hutter, C. Kuhn, P. Rohr, Particle image velocimetry in a foam-like porous structure using refractive index matching: a method to characterize de hydrodynamic performance of porous structures, Experiments in fluids 53 (2012) 1123-1132.

[42] B. Armaly, F. Durst, J. Pereira, B. Shonung, Experimental and theoretical investigation of backward-facing step flow, Journal of Fluid Mechanics 127 (1983) 473-496.

[43] J. Kostas, J. Soria, M. Chong, A study of a backward facing step flow at two Reynolds numbers, 14th Australasian Fluid Mechanics Conference (2001) 609-612.

[44] C. Tropea, R. Gackstatter, The flow over two-dimensional surface-mounted obstacles at low Reynolds number, Journal of Fluids Engineering 107 (1985) 489-494.

[45] F. Durst, M. Founti, S. Obi, Experimental and computational investigation of the two-dimensional channel flow over two feces in tandem, Journal of Fluids Engineering 110 (1988) 48-54.

[46] I. Celik, Z. Cehreli, I. Yavuz, Index of Resolution Quality for Large Eddy Simulations, Journal of Fluids Engineering 127 (2005) 949-958.

[47] A. Lucius, G. Brenner, Numerical Simulation and Evaluation of Velocity Fluctuations During Rotating Stall of a Centrifugal Pump, Journal of Fluids Engineering 133 (2011) 081102-1 - 081102-8.

[48] L. Konnigk, B. Torner, F. Wurm, Application of verification methods on a complex flow field calculated by Large Eddy Simulation: Blood pump flow, 7th European Conference on Computational Fluid Dynamics (2018) 1 - 13.

[49] A. Dastbelaraki, M. Yaghoubi, M. Tavakol, A. Rahmatmand, Numerical analysis of convection heat transfer from an array of perforated fins using RANS and LES method, Applied Mathematical Modelling .

[50] K. Frampton, The effect of flow-induced coupling on sound radiation from convected fluid loaded plates, The Journal of the Acoustical Society of America 117 (2005) 1129-1137.

[51] M. Pastor, M. Binda, T. Harcarik, Modal Assurance Criterion, Procedia Engineering 48 (2012) 543-548.

[52] H. Werner, H. Wengle, Large-eddy simulation of turbulent flow over a square rib in a channel, Advances in Turbulence 2 (1989) $418-423$.

[53] S. Schmidt, F. Thiele, Comparison of numerical methods applied to the flow over wall-mounted cubes, International Journal of Heat and Fluid Flow 23 (2002) 330-339.

[54] M. Ariff, S. Salim, S. Cheah, Wall y+ approach for dealing with turbulent flow over a surface mounted cube: Part 2-High Reynolds number, Proceedings of 7th International Conference on CFD in the Minerals and Process Industries CSIRO, Melbourne, Australia

[55] A. K. Chopra, Modal analysis of linear dynamic systems: physical interpretation, Journal of structural engineering 122 (1996) $517-527$. 


\begin{tabular}{|c|c|}
\hline$L_{r e f}$ & Length of the obstacle face. Reference length \\
\hline$\rho_{\infty}$ & Density of the fluid \\
\hline$\mu$ & Viscosity of the fluid \\
\hline$V_{\infty}$ & Inlet velocity magnitude \\
\hline $\operatorname{Re}$ & Reynolds number \\
\hline$M a$ & Mach number \\
\hline$E$ & Young's Modulus \\
\hline$\rho_{s}$ & Density of the solid \\
\hline$v$ & Poisson's Ratio \\
\hline$h$ & Plate thickness \\
\hline$\vec{x}=\{x, y, z\}$ & Position vector \\
\hline$t$ & Time \\
\hline$p$ & Static pressure \\
\hline$u$ & Displacement of the plate \\
\hline$k^{*}$ & Stiffness parameter \\
\hline$m^{*}$ & Mass parameter \\
\hline$f$ & Frequency \\
\hline St & Strouhal number \\
\hline$C_{p}$ & Pressure coefficient \\
\hline$\vec{V}=\left\{V_{x}, V_{y}, V_{z}\right\}$ & Fluid velocity \\
\hline$\tau_{i j}$ & ij component of the fluid stress tensor \\
\hline$\tau_{t}$ & Turbulent stress tensor \\
\hline$\mu_{t}$ & Turbulent viscosity \\
\hline $\mathbf{S}$ & Fluid strain tensor \\
\hline $\mathbf{I}$ & Identity matrix \\
\hline$k$ & Turbulent kinetic energy \\
\hline $\mathbf{S}$ & Deformation parameter \\
\hline$\Delta$ & LES length scale parameter \\
\hline$C_{w}$ & LES model coefficient \\
\hline $\mathcal{V}$ & Cell volume \\
\hline$C F L$ & Courant number \\
\hline$\varepsilon$ & Specific turbulent dissipation \\
\hline$C_{\mu}$ & RANS model constant \\
\hline$x_{\text {reattach }}$ & Location of the reattachment \\
\hline$\eta$ & Calculated to total turbulent kinetic energy ratio \\
\hline$k_{c}$ & Calculated turbulent kinetic energy \\
\hline$k_{S G S}$ & Modeled subgrid scale turbulent kinetic energy \\
\hline$F_{x}$ & Force exerted over the step on the $x$ direction \\
\hline$F_{y}$ & Force exerted over the step on the $y$ direction \\
\hline$C_{L}$ & Lift coefficient \\
\hline$C_{D}$ & Drag coefficient \\
\hline$A$ & Area \\
\hline$\phi_{i}$ & Modal form \\
\hline
\end{tabular}

\section{List of Figures}

Figure 1 Diagram of the working flow used during the current work . . . . . . . . . . . . 3

Figure 2 Fluid domain geometry sketch $($ not scale) $\ldots \ldots \ldots \ldots \ldots$

Figure 3 Ratio of CFL distribution over the entire fluid domain for the meshes of $N_{\text {elements }} \approx 4 \times 10^{6}$ (left) and $N_{\text {elements }} \approx 20 \times 10^{6}$ (right) $\ldots \ldots \ldots \ldots \ldots \ldots \ldots \ldots$

Figure 4 Sketch of the computational fluid mesh . . . . . . . . . . . . . . . . . . 7

Figure 5 Pressure coefficient distribution at the back plate for: fluid finite volume mesh (left) and structural finite element mesh (right) at an arbitrary time step of the Large Eddy Simulation solution . . . . 
Figure 6 Force time history (left) and frequency content (right). Computation for the mesh with $N_{\text {elements }} \approx$

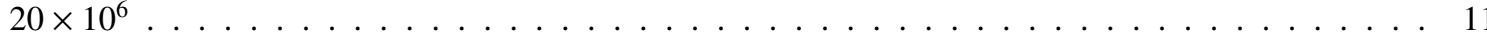

Figure 7 Vectors of time averaged velocity for the identification of the experimetnal reattachment length 11

Figure 8 Time averaged velocity using LES (top) and RANS (bottom) for the mesh with $N_{\text {elements }} \approx 20 \times 10^{6} \quad 12$

Figure 9 Average non dimensional wall shear stress after the step (left) and over the step (right) $N_{\text {elements }} \approx$ $20 \times 10^{6}$ and identification of the reatahcment location . . . . . . . . . . . . . . 12

Figure 10 Turbulent viscosity ratio for the RANS computation with the mesh of $N_{\text {elements }} \approx 20 \times 10^{6} \ldots .13$

Figure 11 Resolved (top), subgrid scale (middle) and total turbulent kinetic energy at the midplane. Computation for the mesh with $N_{\text {elements }} \approx 4 \times 10^{6} \ldots \ldots \ldots \ldots \ldots \ldots \ldots \ldots \ldots$

Figure 12 Ratio of resolved over total turbulent kinetic energy at the midplane. Computation for the mesh with $N_{\text {elements }} \approx 4 \times 10^{6} \ldots \ldots \ldots \ldots \ldots \ldots$. . . . . . . . . . . . . . . . . . . . . . 14

Figure 13 Distribution of the ratio of resolved over total turbulent kinetic energy and wall $y^{+}$. Computation for the mesh with $N_{\text {elements }} \approx 4 \times 10^{6} \ldots \ldots \ldots \ldots \ldots \ldots \ldots \ldots \ldots$

Figure 14 Velocity time history (left) and frequency content (right) at a point $P 1$, located near to the main shear layer. Computations for the mesh with $N_{\text {elements }} \approx 20 \times 10^{6} \ldots \ldots$. . . . . . . . . . . . . . 15

Figure 15 Visualization of turbulent strucures at an arbitrary instant for the computation with $N_{\text {elements }} \approx$ $20 \times 10^{6}$. Contour of instantaneous velocity field at the midplane (top) and volumetric render of the low velocity at the wake $($ bottom $) \ldots \ldots \ldots \ldots \ldots \ldots$

Figure 16 Energy spectra at the point located near to the shear layer, $P 1$ (left) and frequency content of the pressure coefficient at a point located over the plate, at point G5. Comparison between different

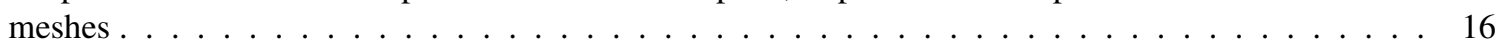

Figure 17 Calculated modal displacements . . . . . . . . . . . . . . . . . . . . 17

Figure 18 Modal Assurance Criterion matrix _. . . . . . . . . . . . . . . . . . . . . . 18

Figure 19 Time averaged pressure coefficient (left) and mean line plate displacement (right) after the step. Comparison between LES and RANS computations using different meshes . . . . . . . . . . . . . . . 18

Figure 20 Time averaged displacement field with LES (top) and RANS (bottom) computations. Computation with the mesh of $N_{\text {elements }} \approx 20 \times 10^{6} \ldots \ldots \ldots \ldots \ldots \ldots \ldots \ldots$

Figure 21 Time history (left) and frequency content (right) of the unsteady pressure coefficient at points located over the plate for the mesh of $N_{\text {elements }} \approx 20 \times 10^{6} \ldots \ldots \ldots \ldots$. . . . . . . . . 20

Figure 22 Frequency response of the plate displacement under the action of the turbulent pressure field at different frequencies . . . . . . . . . . . . . . . . . . . . . . . . 21

Figure 23 Modal contribution of each eigenvector to the total displacement of the plate under the action of the turbulent pressure field at different frequencies. Only the first 7 modes are shown . . . . . . . . 22

Figure 24 Mean displacement spectra for the current calculation (left) and collection of the displacements results taken from Schafer et al. for a plate of thickness $h=40 \mu \mathrm{m}$ (right) . . . . . . . . . . . 23 